\title{
Cosmological constant, near brane behavior and singularities
}

\author{
Fridrik Freyr Gautason, ${ }^{a}$ Daniel Junghans ${ }^{a, b}$ and Marco Zagermann ${ }^{a}$ \\ ${ }^{a}$ Institut für Theoretische Physik \& Center for Quantum Engineering and Spacetime Research, \\ Leibniz Universität Hannover, \\ Appelstraße 2, 30167 Hannover, Germany \\ ${ }^{b}$ Department of Physics 8 Institute for Advanced Study, \\ Hong Kong University of Science and Technology, \\ Clear Water Bay, Kowloon, Hong Kong \\ E-mail: fridrik.gautason@itp.uni-hannover.de, \\ daniel.junghans@itp.uni-hannover.de, \\ marco.zagermann@itp.uni-hannover.de
}

ABSTRACT: We show that the classical cosmological constant in type II flux compactifications can be written as a sum of terms from the action of localized sources plus a specific contribution from non-trivial background fluxes. Exploiting two global scaling symmetries of the classical supergravity action, we find that the flux contribution can in many interesting cases be set to zero such that the cosmological constant is fully determined by the boundary conditions of the fields in the near-source region. This generalizes and makes more explicit previous arguments in the literature. We then discuss the problem of putting $\overline{\mathrm{D} 3}$-branes at the tip of the Klebanov-Strassler throat glued to a compact space in type IIB string theory so as to engineer a de Sitter solution. Our result for the cosmological constant and a simple global argument indicate that inserting a fully localized and backreacting $\overline{\mathrm{D} 3}$ brane into such a background yields a singular energy density for the NSNS and RR 3-form field strengths at the $\overline{\mathrm{D} 3}$-brane. This argument does not rely on partial smearing of the $\overline{\mathrm{D} 3}$-brane or a linearization of field equations, but on a few general assumptions that we also discuss carefully.

Keywords: Flux compactifications, dS vacua in string theory, D-branes, Superstring Vacua

ARXIV EPRINT: 1301.5647 


\section{Contents}

1 Introduction $\quad 1$

2 Type II supergravity 4

3 The cosmological constant as a sum of source terms $\quad 6$

$\begin{array}{lll}3.1 & \text { Two scaling symmetries } & 6\end{array}$

3.2 The method 8

$\begin{array}{lll}3.3 & \text { On-shell action and cosmological constant } & 10\end{array}$

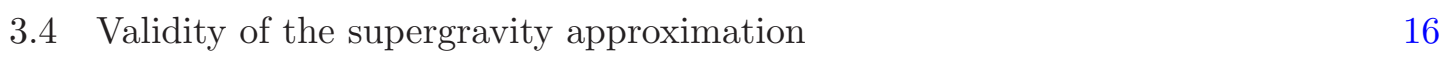

$\begin{array}{lll}4 & \text { Examples } & 17\end{array}$

$\begin{array}{lll}4.1 & \text { The GKP solutions } & 17\end{array}$

$4.2 \overline{\mathrm{D} 6}$-branes on $\mathrm{AdS}_{7} \times S^{3} \quad 18$

$\begin{array}{lll}4.3 & \mathrm{SU}(3) \text {-structure manifolds with O6-planes } & 19\end{array}$

$\begin{array}{ll}4.4 \text { The DGKT solutions } & 20\end{array}$

5 Singular $\overline{\mathrm{D} 3}$-branes in the Klebanov-Strassler throat $\quad 21$

$\begin{array}{lll}5.1 \text { Ansatz } & 21\end{array}$

$\begin{array}{ll}5.2 & \text { The argument } 23\end{array}$

$\begin{array}{llr}6 & \text { Conclusion } & 27\end{array}$

A Explicit manipulations of the equations of motion 28

\section{Introduction}

A better understanding of string compactifications involving localized sources such as Dbranes or orientifold planes is an important task for string phenomenology. Unfortunately, for most scenarios a full solution to the ten-dimensional equations of motion seems to be out of reach, even in the supergravity approximation, because the involved differential equations are too complex. On the other hand, commonly used procedures for simplifying this task, such as a smearing of the localized sources over the compact space, may introduce their own problems and need not necessarily capture essential features of the true solution (see e.g. [1-3]). It would therefore be desirable to be able to compute important observables such as the cosmological constant without having to know the full ten-dimensional dynamics or rely on simplifications such as smearing.

In the first part of this work, we will show, in the context of type II supergravity coupled to D-branes and O-planes, that such a method often exists, building upon previous work that had already pointed towards this possibility [4-6]. In particular, we will argue that 
the cosmological constant, $\Lambda$, can often be expressed as a sum of terms that are due to the action of localized sources,

$$
\Lambda \propto \sum_{p} c_{p}\left(S_{\mathrm{DBI}}^{(p)}+S_{\mathrm{CS}}^{(p)}\right)
$$

where $S_{\mathrm{DBI}}^{(p)}$ and $S_{\mathrm{CS}}^{(p)}$ are the on-shell evaluated DBI and Chern-Simons actions of the D $p$ branes and/or $\mathrm{O} p$-planes present in the corresponding supergravity solution, and $c_{p}$ are $p$-dependent constants. Thus, in compactification scenarios where our reasoning holds, $\Lambda$ is entirely specified by the classical boundary conditions of some of the bulk fields at the positions of the sources and independent of the details of the ten-dimensional bulk dynamics.

Such a property was noticed before in $[4,5]$. Using a single scaling symmetry of the action of different supergravity theories, the authors were able to relate $\Lambda$ to boundary terms involving the supergravity fields that have to be evaluated in the near-source region. They pointed out, however, that topologically nontrivial background fluxes can also give contributions that arise from the patching of gauge charts. The explicit evaluation of these subtle flux and all source contributions together with their gauge dependence for a general type II compactification make up the first part of our paper. Furthermore, we show that the flux contributions actually vanish in many interesting examples such that the only contributions to the cosmological constant are due to the action of D-branes and O-planes.

From a somewhat different angle, also the results of [6] suggested such a behavior. There, it was shown that the cosmological constant in solutions of perturbative heterotic string theory is zero to all orders in $\alpha^{\prime}$, unless one introduces spacetime-filling fluxes or considers string loop or non-perturbative corrections. Since the argument only used the scaling properties of the effective potential with respect to the dilaton, it was then conjectured that a similar reasoning should also be applicable for the type II string, with the exception that then also D-brane and O-plane sources should give a contribution to $\Lambda$. For classical solutions of type II supergravity, this suggests that, in absence of spacetime-filling flux, any non-zero contribution to $\Lambda$ must be generated by terms that are due to localized sources. It turns out, however, that the intuitive scaling argument of [6] is complicated in the type II string by a subtlety related to the RR fields: in a frame, where the bulk action scales uniformly with the dilaton, non-trivial couplings of the RR potentials with derivatives of the dilaton of the form $\mathrm{d} \phi \wedge C \wedge F$ arise. These couplings are only present in the type II string, not in the heterotic string. In the presence of background fluxes, they can be shown to yield non-zero contributions to $\Lambda$, thus spoiling the argument sketched above.

As we will show in this paper, however, it remains true in many cases that $\Lambda$ is completely determined by a sum of source terms. The reason is that classical type II (and also heterotic) supergravity exhibits a two-parameter scaling symmetry, related to the dilaton scaling and the mass scaling of the classical action $[7,8]$. Both the scaling symmetry exploited in [5] and the one implicitly used in [6] are special cases of this more general symmetry. As we will show below, it ensures that one can often find a particular combination of the equations of motion such that all bulk terms are eliminated from the equation determining $\Lambda$, leaving a contribution entirely from localized sources. The cosmological constant is then indeed given by a sum of source terms as initially claimed. More precisely, this 
can be shown to hold for maximally symmetric compactifications of type II supergravity involving sources of arbitrary dimension and at most $H$ flux and one type of RR flux.

In the second part of this work, we discuss an application of our result to the idea of placing D3-branes at the bottom of the Klebanov-Strassler solution [9-11], a setup that has been suggested for the construction of meta-stable de Sitter vacua in string theory starting with [12]. The backreaction of $\overline{\mathrm{D} 3}$-branes on the Klebanov-Strassler geometry has recently been subject of intense discussions [13-23]. Part of this debate concerns the computational evidence for a singularity in fields that do not directly couple to the anti-branes as it emerged in several approaches.

More precisely, the presence of this singularity has so far been demonstrated in simplified setups that use certain approximations. In earlier works on the subject, this involved a partial smearing of the branes and a linearization of the equations of motion around the Klebanov-Strassler background [14-17]. [18] therefore also discusses the possibility that the singularity might just be an artifact of perturbation theory and disappear in the full setup (see however [19]). Although it could recently be shown in [20] that also the non-linear equations of motion necessarily lead to a singular solution, the analysis still required partially smeared branes. An analysis of the fully localized case could only be carried out for a simplified toy model with $\overline{\mathrm{D} 6}$-branes [3, 24, 25], which is related by T-duality to partially smeared $\overline{\mathrm{D} 3}$-branes on $\mathbb{R}^{3} \times T^{3}[19]$. In this simplified setup, it was shown that fully localized branes in a non-BPS flux background lead to a singularity in the energy density of the $H$ flux, which is not directly sourced by the $\overline{\mathrm{D} 6}$-branes.

As our result from the first part of the paper relates the near-brane behavior of the supergravity fields to the effective cosmological constant, it is natural to try to apply this to $\overline{\mathrm{D} 3}$-branes in the KS background. We show that under a few assumptions this would indeed be possible and confirm the presence of a non-standard singularity at the $\overline{\mathrm{D} 3}$-brane similar to the one discussed before, but now without the approximation of any smearing and by using the full non-linear supergravity equations.

This paper is organized as follows. In section 2, we establish our notation and conventions and state the equations of motion of type II supergravity used in the following sections. In section 3, we discuss the two scaling symmetries of classical type II supergravity. We then show that the cosmological constant can be written as a sum of source terms and a term involving topological background fluxes that can in many cases be gauged away by exploiting a combination of the symmetries. In section 4, we present several explicit examples of compactifications of type II supergravity and show how our framework can be applied to them in order to obtain an expression for the cosmological constant in terms of the actions of localized sources. In section 5 , we consider the backreaction of $\overline{\mathrm{D} 3}$-branes on the Klebanov-Strassler throat glued to a compact space in type IIB string theory and discuss under what assumptions our previous results would imply the existence of a singularity in the energy densities of $H$ and $F_{3}$. We conclude with some comments in section 6 . 


\section{Type II supergravity}

We start by establishing our notation and conventions. ${ }^{1}$ In the tree-level supergravity approximation, the low energy effective action of type II string theory in Einstein frame can be written as

$$
S=S_{\mathrm{bulk}}+S_{\mathrm{loc}}
$$

with

$$
S_{\text {bulk }}=S_{\text {NSNS }}+S_{\mathrm{RR}}=\int \star_{10}\left\{R-\frac{1}{2}|\mathrm{~d} \phi|^{2}-\frac{1}{2} \mathrm{e}^{-\phi}|H|^{2}-\frac{1}{4} \sum_{n} \mathrm{e}^{\frac{5-n}{2} \phi}\left|F_{n}\right|^{2}\right\} .
$$

Here, $R$ is the curvature scalar of the metric $g, \star_{10}$ denotes the ten-dimensional Hodge operator associated with $g, \phi$ is the dilaton, $H$ is the NSNS 3-form field strength, and $F_{n}$ are the RR field strengths. For an $n$-form $A$, the norm $|A|^{2}$ is defined by

$$
|A|^{2} \star_{10} 1=\frac{1}{n !} A_{\mu_{1} \ldots \mu_{n}} A^{\mu_{1} \ldots \mu_{n}} \star_{10} 1=\left(\star_{10} A\right) \wedge A .
$$

We often consider warped product spaces $\mathcal{M}^{(10)}=\mathcal{M}^{(d)} \times_{w} \mathcal{M}^{(k)}$, where $\star_{d}$ and $\star_{k}$ then denote the Hodge operators of the corresponding warped metric factors. For factorizing forms $A_{p} \wedge B_{q}$, where $A_{p}$ is a $p$-form on $\mathcal{M}^{(d)}$ and $B_{q}$ a $q$-form on $\mathcal{M}^{(k)}$, these Hodge operators satisfy the useful identity $\star_{10}\left(A_{p} \wedge B_{q}\right)=(-1)^{p(k-q)}\left(\star_{d} A_{p}\right) \wedge\left(\star_{k} B_{q}\right)$. In general, we have $\left(\star_{D}\right)^{2} A_{p}=(-1)^{p(D-p)+t} A_{p}$ for any $p$-form on a $D$-dimensional manifold with $t$ timelike directions.

Throughout this paper, we use the democratic formulation [27], so that the sum over the RR field strengths in (2.2) also includes the dual fields with $n>5$. The field strengths are related to one another by the duality relations

$$
\mathrm{e}^{\frac{5-n}{2} \phi} F_{n}=\star_{10} \sigma\left(F_{10-n}\right),
$$

which have to be imposed on-shell. The operator $\sigma$ here acts on an $n$-form $\omega_{n}$ like

$$
\sigma\left(\omega_{n}\right)=(-1)^{\frac{n(n-1)}{2}} \omega_{n} .
$$

Also notice that, in $(2.2)$, we have set $2 \kappa_{10}^{2}=1$, so that the Planck mass has been absorbed into the definition of the metric.

The term $S_{\text {loc }}$ denotes the action of localized sources corresponding to either $\mathrm{D} p$-branes or $\mathrm{O} p$-planes and reads ${ }^{2}$

$$
S_{\mathrm{loc}}=\sum_{p} S_{\mathrm{loc}}^{(p)}=\sum_{p}\left(S_{\mathrm{DBI}}^{(p)}+S_{\mathrm{CS}}^{(p)}\right)
$$

\footnotetext{
${ }^{1}$ We use the conventions of [26] except that the sign of $B$ is flipped.

${ }^{2}$ We do not include the NSNS 2-form in the DBI action here, because in all the examples we discuss in detail the sources are either point-like in the internal space or they are wrapped O-planes, so that a $B$-field along the world volume cannot occur. Likewise we do not consider D-branes with world volume fluxes in our examples and hence also omit them in the DBI action. It is easy to check that omitting the NSNS 2-form in the DBI action does not lead to a missing term in the $H$-equation of motion, because $\delta S_{D B I} / \delta B_{\mu \nu}$ also vanishes if $B$ and $F$ are set to zero after the field equations are derived (cf. also the explicit expressions in [28]).
} 
with

$$
S_{\mathrm{DBI}}^{(p)}=\mp \mu_{p} \int \star_{p+1} \mathrm{e}^{\frac{p-3}{4} \phi} \wedge \sigma\left(\delta_{9-p}\right), \quad S_{\mathrm{CS}}^{(p)}=\left\{\begin{array}{l}
+\mu_{p} \int\left\langle C \wedge \mathrm{e}^{-B}\right\rangle_{p+1} \wedge \sigma\left(\delta_{9-p}\right) \\
-\mu_{p} \int C_{p+1} \wedge \sigma\left(\delta_{9-p}\right)
\end{array},\right.
$$

where the upper line is for $\mathrm{D} p$-branes and the lower line for $\mathrm{O} p$-planes, and $\mu_{p}>0$ is the absolute value of the $\mathrm{D} p$-brane/O $p$-plane charge. For $\overline{\mathrm{D} p}$-branes and $\overline{\mathrm{O} p}$-planes, the Chern-Simons terms would have the opposite sign. $\star_{p+1}$ is the Hodge operator on the $(p+1)$ dimensional world volume, $\Sigma$, of the source in question, and we define $\delta_{9-p}=\sigma\left(\star_{9-p} 1\right) \delta(\Sigma)$, where $\star_{9-p} 1$ is the $(9-p)$-dimensional volume form transverse to the source (defined such that $\left.\star_{10} 1=\star_{p+1} 1 \wedge \star_{9-p} 1\right)$ and $\delta(\Sigma)$ is the delta distribution with support on $\Sigma$. We also use the polyform notation in (2.7), i. e. $C=\sum_{n} C_{n-1}$ denotes the sum of all electric and magnetic RR potentials that appear in type IIA or type IIB supergravity, and $\mathrm{e}^{-B}$ is defined as a power series of wedge products. The symbol $\langle\cdots\rangle_{p+1}$ denotes a projection to the form degree $p+1$, i.e.,

$$
\left\langle C \wedge \mathrm{e}^{-B}\right\rangle_{p+1}=C_{p+1}-C_{p-1} \wedge B+\frac{1}{2} C_{p-3} \wedge B \wedge B-\ldots
$$

Throughout this paper, we restrict ourselves to warped compactifications to $d \geq 4$ dimensions that preserve maximal symmetry in the non-compact $d$-dimensional spacetime. Accordingly, we only consider spacetime-filling sources extending in $p+1 \geq d$ dimensions. Furthermore, all fields are assumed to depend only on the internal coordinates $x^{m}$. The form fields are allowed to have legs in external directions only if they are spacetime-filling, in other words they have to be of rank $d$ or higher. All other form fields are purely internal. We assume a warped metric of the form

$$
\mathrm{d} s_{10}^{2}=g_{\mu \nu} \mathrm{d} x^{\mu} \mathrm{d} x^{\nu}+g_{m n} \mathrm{~d} x^{m} \mathrm{~d} x^{n}, \quad g_{\mu \nu}=\mathrm{e}^{2 A} \tilde{g}_{\mu \nu},
$$

where $A$ is the warp factor and $\tilde{g}_{\mu \nu}$ is the unwarped $d$-dimensional metric corresponding to a Minkowski or (A)dS spacetime. We will also put a tilde on quantities such as Hodge operators, covariant derivatives or contractions of tensors if they are constructed using the unwarped metric instead of the warped one.

We now list the relevant equations of motion. The trace of the external Einstein equation reads

$$
R_{d}=\frac{d}{2}\left(\mathcal{L}-\sum_{p} \mathcal{L}_{\mathrm{CS}}^{(p)}\right)+\frac{d}{4} \sum_{n} \mathrm{e}^{\frac{5-n}{2} \phi}\left|F_{n}^{\mathrm{ext}}\right|^{2},
$$

where $R_{d}=R_{\mu \nu} g^{\mu \nu}$ is the $d$-dimensional Ricci scalar and we denote the spacetime-filling RR field strengths by $F_{n}^{\text {ext }} . \mathcal{L}$ is the Lagrangian including all bulk terms and the DBI and CS terms due to the localized sources, and $\mathcal{L}_{\mathrm{CS}}^{(p)}$ are the CS parts of the source Lagrangian. For the warped metric (2.9), one finds

$$
R_{d}=\frac{2 d}{d-2} \mathrm{e}^{-2 A} \Lambda-\mathrm{e}^{-d A} \tilde{\nabla}^{2} \mathrm{e}^{d A}
$$


where $\Lambda$ is the $d$-dimensional cosmological constant. Substituting this into (2.10) and integrating over ten-dimensional spacetime then yields

$$
\frac{8 v \mathcal{V}}{d-2} \Lambda=2\left(S-\sum_{p} S_{\mathrm{CS}}^{(p)}\right)+\sum_{n} \int \star_{10} \mathrm{e}^{\frac{5-n}{2} \phi}\left|F_{n}^{\text {ext }}\right|^{2},
$$

where we have introduced the volume factors

$$
v=\int \tilde{\star}_{d} 1, \quad \mathcal{V}=\int \star_{10-d} \mathrm{e}^{(d-2) A} .
$$

The Bianchi identities for the RR fields are

$$
\mathrm{d}_{-H} F+j=0,
$$

where $F=\sum_{n} F_{n}$ is the polyform containing the sum over all RR field strengths, $\mathrm{d}_{-H}=$ $\mathrm{d}-H \wedge$ is the twisted exterior derivative, and $j$ is the polyform containing the sum over all source contributions of the different Bianchi identities, where $j=\sum_{p} \mu_{p}\left\langle\delta \wedge \mathrm{e}^{B}\right\rangle_{9-p}$ for D-branes and $j=-\sum_{p} \mu_{p} \delta_{9-p}$ for O-planes. Finally, we state the dilaton equation,

$$
\nabla^{2} \phi=-\frac{1}{2} \mathrm{e}^{-\phi}|H|^{2}+\sum_{n} \frac{5-n}{8} \mathrm{e}^{\frac{5-n}{2} \phi}\left|F_{n}\right|^{2} \pm \sum_{p} \frac{p-3}{4} \mu_{p} \mathrm{e}^{\frac{p-3}{4} \phi} \delta(\Sigma)
$$

and the equation of motion and Bianchi identity for $H$,

$$
\mathrm{d}\left(\mathrm{e}^{-\phi} \star_{10} H\right)-\frac{1}{2}\langle F \wedge \sigma(F)\rangle_{8}=0, \quad \mathrm{~d} H=0 .
$$

\section{The cosmological constant as a sum of source terms}

In this section, we will introduce two independent scaling symmetries satisfied by the action (2.1) and use them to derive an expression for the cosmological constant $\Lambda$ in terms of the (on-shell evaluated) action of localized sources.

\subsection{Two scaling symmetries}

It is known since the 1980s [7] that the terms in the low energy effective action of string theory must satisfy simple scaling properties when the dilaton or equivalently the string coupling constant is scaled. This property is inherited from the simple coupling of the dilaton to the world sheet curvature in string perturbation theory and is manifest in the string frame of the 10D effective action. In Einstein frame, the scaling does not only affect the dilaton $\phi$, but also the metric $g_{M N}$ and the $\mathrm{RR}(n-1)$-forms $C_{n-1}$ of the type II theories:

$$
\mathrm{e}^{-\phi} \mapsto s \mathrm{e}^{-\phi}, \quad g_{M N} \mapsto \sqrt{s} g_{M N}, \quad C_{n-1} \mapsto s C_{n-1},
$$

where $s$ is a scaling parameter. This then leads to

$$
S^{(\chi)} \mapsto s^{\chi} S^{(\chi)},
$$


where $\chi$ is the Euler characteristic of the world sheet from which the contribution, $S^{(\chi)}$, to the effective action was derived. For a standard low energy effective action consisting of the classical two-derivative action for the bulk supergravity fields, $S_{\text {bulk }}$, and the lowest order action due to the presence of localized sources, $S_{\text {loc }}$, we then get

$$
S=S_{\mathrm{bulk}}+S_{\mathrm{loc}} \mapsto s^{2} S_{\mathrm{bulk}}+s S_{\mathrm{loc}}
$$

This can be verified using (3.1) in (2.2) and (2.7) or simply by using the corresponding actions in the string frame. Thus, in absence of localized sources, the effect of (3.1) is to rescale the tree-level supergravity action by an overall factor $s^{2}$. The transformations (3.1) are then a symmetry of the theory, since they leave the equations of motion invariant.

A second scaling symmetry [8] can be obtained from the mass dimension of the fields, which can be determined from the fact that the effective action is a derivative expansion and has mass dimension zero. Using that the mass dimension of the coordinates is -1 and the mass dimension of a derivative is +1 , one can count the number of derivatives of a given term in the action and the number of dimensions that are integrated over to determine the mass dimension of the fields. If one then scales the fields in the effective action according to their mass dimension but leaves the coordinates unscaled, one obtains a non-trivial scaling of the terms in the action. The corresponding scaling of the bosonic fields in type II string theory is ${ }^{3}$

$$
g_{M N} \mapsto t^{-2} g_{M N}, \quad C_{n-1} \mapsto t^{-(n-1)} C_{n-1}, \quad B \mapsto t^{-2} B,
$$

where $t$ is another scaling parameter. This yields the following scaling of the terms in the low energy action

$$
S_{i}^{D} \mapsto t^{i-D} S_{i}^{D}
$$

where $D$ denotes the number of dimensions that are integrated over (usually $D=10$, but $D$ is less than ten for source terms) and $i$ denotes the number of derivatives of the terms involved. For a two-derivative bulk action and zero-derivative source terms with $(p+1)$-dimensional world volume, we thus get

$$
S=S_{\mathrm{bulk}}+S_{\mathrm{loc}} \mapsto t^{-8} S_{\mathrm{bulk}}+\sum_{p} t^{-p-1} S_{\mathrm{loc}}^{(p)}
$$

as can be verified using (3.4) in (2.2) and (2.7). In absence of localized sources, the transformations (3.4) are a symmetry, since they rescale the bulk action by an overall factor $t^{-8}$ and thus leave the equations of motion invariant. Together with (3.3), this implies that the type II supergravity action at tree-level has two global scaling symmetries, which are explicitly broken by terms that are due to the presence of localized sources.

\footnotetext{
${ }^{3}$ This symmetry is sometimes called Trombone symmetry in the context of supergravity, see for example [29]. Note that in our conventions the exponent of $t$ in (3.4) actually corresponds to the length (i.e. the inverse mass) dimension of the field.
} 


\subsection{The method}

As mentioned in the introduction, the above scaling symmetries can often be used to derive an expression for the cosmological constant $\Lambda$ in terms of the on-shell action of localized sources. In those cases where this is possible, $\Lambda$ is thus determined by the boundary conditions of some of the bulk supergravity fields at the positions of the sources and independent of the details of the dynamics in the bulk. We will argue below that this is possible for compactifications that involve at most NSNS $H$ flux and not more than one type of RR flux. This extends the recent work [5] by an explicit evaluation of the general flux contribution and the use of a second scaling symmetry to gauge them away in the above-mentioned cases. The resulting simplified expression for the cosmological constant in terms of on-shell brane and O-plane actions can then be applied to several interesting type II flux compactifications, as we discuss in the remainder of the paper.

The strategy for deriving our expression for $\Lambda$ is as follows. At first, the scaling symmetries are used to derive an expression for the action (2.1) that holds on-shell. This on-shell expression can then be substituted into the integrated Einstein equation (2.12), which, as we will show, eliminates the dependence of the equation on the bulk fields up to certain flux terms and yields the desired result for $\Lambda$. Before we discuss how to derive the on-shell action in the general case, let us at first review the basic principle [5] using a simple example. Consider an action $S\left[\psi_{i}\right]$ that depends on a number of fields $\psi_{i}$ and that satisfies a scaling symmetry,

$$
S\left[\tau^{k_{i}} \psi_{i}\right]=\tau^{k} S\left[\psi_{i}\right]
$$

where the scaling parameter $\tau$ is a real number, and $k$ is assumed to be non-vanishing. We can then take the $\tau$ derivative of (3.7) to obtain

$$
\int \sum_{i} k_{i} \tau^{k_{i}-1} \psi_{i} \frac{\delta S\left[\tau^{k_{i}} \psi_{i}\right]}{\delta\left(\tau^{k_{i}} \psi_{i}\right)}=k \tau^{k-1} S\left[\psi_{i}\right],
$$

where we have written the result in terms of the the usual functional derivative (which for derivative terms implicitly involves partial integrations). Evaluating the equation at $\tau=1$ and using the fact that the fields satisfy the equations of motion $\delta S\left[\psi_{i}\right] / \delta \psi_{i}=0$, we then find that the left-hand side of (3.8) vanishes and

$$
S\left[\psi_{i}\right]=0
$$

on-shell.

In deriving (3.9), however, we made two simplifications that do in general not hold in the context of string compactifications. The right-hand side of the equation is therefore often more complicated than in this simple example. First, we assumed that all terms in the action $S\left[\psi_{i}\right]$ scale uniformly with $\tau$. When we identify $\tau$ with the scaling parameters $s$ and $t$ of the previous subsection, this is then not true in string theory when localized sources are included, as can be seen from (3.3) and (3.6). ${ }^{4}$ Second, when we evaluated $\mathrm{d} S\left[\tau^{k_{i}} \psi_{i}\right] / \mathrm{d} \tau$ to arrive at (3.8), we had to integrate by parts all those terms in $S\left[\tau^{k_{i}} \psi_{i}\right]$

\footnotetext{
${ }^{4}$ The assumption would also break down if one includes, e.g., $\alpha^{\prime}$ or loop corrections.
} 
that involve derivatives of $\psi_{i}$. In string theory, however, many compactifications involve the presence of non-trivial background fluxes. The corresponding NSNS and/or RR field strength(s) then have a non-exact part such that, globally, they cannot be written in terms of a gauge potential. Instead, their gauge potentials are only locally defined. Thus, total derivatives involving the NSNS or RR gauge potentials do not necessarily integrate to zero anymore but may involve non-trivial contributions from patches of different gauge charts, which would yield an extra contribution when one integrates by parts. When we repeat the above calculation for the general action (2.1), we therefore expect that the right-hand side of (3.9) receives two contributions: one contribution due to the presence of localized sources and another one due to non-trivial background fluxes.

In order to account for the possibility of flux, we explicitly divide the NSNS and RR field strengths into a flux part, which is closed but not exact, and a fluctuation, which is exact and given in terms of a globally defined gauge potential. For $H$, we thus write

$$
H=\mathrm{d} B+H^{b},
$$

where $H^{b}$ denotes the background flux and $B$ is the fluctuating globally defined NSNS potential. Since $H^{b}$ is closed, the Bianchi identity $\mathrm{d} H=0$ is satisfied such that our definition is consistent. ${ }^{5}$

For the RR field strengths, separating off the non-exact part is more subtle. This is related to the fact that their Bianchi identities are more complicated and, in particular, that some of them receive contributions from localized sources. Since we only consider spacetime-filling sources in this paper, they enter the Bianchi identities as delta forms whose legs are always in some of the internal directions. Thus, a source term can only show up in the Bianchi identity for the purely internal part of the corresponding RR field strength. It is therefore convenient to split the polyform $F=\sum_{n} F_{n}$ into a part $F^{\text {int }}=\sum_{n} F_{n}^{\text {int }}$, which contains all RR field strengths that are purely internal and may have a source term in their Bianchi identity, and a part $F^{\text {ext }}=\sum_{n} F_{n}^{\text {ext }}$, which contains all RR field strengths that are spacetime-filling (and possibly also have legs in the internal part) and, accordingly, do not have a source term in their Bianchi identity,

$$
F=F^{\mathrm{int}}+F^{\mathrm{ext}} \text {. }
$$

For $F^{\text {ext }}$, the Bianchi identities (2.14) then simplify to

$$
\mathrm{d}_{-H} F^{\mathrm{ext}}=0 .
$$

This allows us to make the ansatz

$$
F^{\mathrm{ext}}=\mathrm{d}_{-H} C^{\mathrm{ext}}+\mathrm{e}^{B} \wedge F^{b},
$$

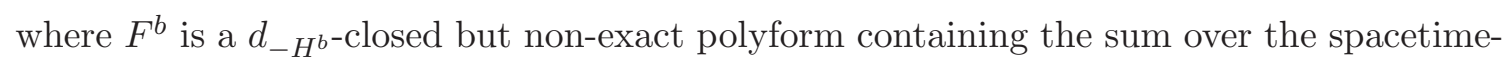
filling background fluxes and $C^{\text {ext }}$ is a polyform containing the sum over the spacetimefilling $\mathrm{RR}$ potentials. In a (maximally symmetric) type IIB compactification to 4 dimensions, for example, we would have $F^{b}=F_{5}^{b}+F_{7}^{b}+F_{9}^{b}$ and $C^{\text {ext }}=C_{4}^{\text {ext }}+C_{6}^{\text {ext }}+C_{8}^{\text {ext }}$, since

\footnotetext{
${ }^{5}$ We do not consider compactifications involving NS5-branes in this paper, i.e. the Bianchi identity for $H$ does not contain a source term.
} 
only forms of rank 4 or higher would be allowed to be spacetime-filling. One can verify that (3.13) solves the Bianchi identities (3.12) and is therefore a consistent ansatz for the field strengths $F^{\text {ext }}$.

The Bianchi identities of the internal field strengths, $F^{\text {int }}$, however, may contain source terms such that these field strengths can in general not be written in a way similar to (3.13) everywhere on the compact space. We will circumvent this problem in this paper by simply expressing, at the level of the equations of motion, $F^{\text {int }}$ in terms of their dual field strengths $F^{\text {ext }}$, which then in turn can be expressed in terms of (3.13). If, for example, $F_{3}=F_{3}^{\text {int }}$ is internal, we can express it in terms of the spacetime-filling $F_{7}=F_{7}^{\text {ext }}$ via the duality relation $F_{3}^{\text {int }}=-\mathrm{e}^{-\phi} \star_{10} F_{7}^{\text {ext }}$ and then use (3.13) to split $F_{7}^{\text {ext }}$ into an exact and a non-exact part. ${ }^{6}$

Finally, let us note that, since we put the non-exact parts of the NSNS and RR field strengths into $H^{b}$ and $F^{b}$, we can assume that the gauge potentials $B$ and $C^{\text {ext }}$ are globally defined. This implies that total derivatives involving $B$ and $C^{\text {ext }}$ integrate to zero on a compact space, which will be used below. It should also be mentioned that, under the scalings (3.1) and (3.4), the flux terms $H^{b}$ and $F^{b}$ behave in the same way as the corresponding gauge potentials do. This follows from the fact that the mass dimension and the coupling to the dilaton is the same for the exact and the non-exact parts of the NSNS and RR field strengths.

\subsection{On-shell action and cosmological constant}

Let us now discuss how to derive the on-shell expression for the action (2.1) that will later be used in the integrated Einstein equation (2.12) to obtain our result for $\Lambda$. Contrary to the simple example sketched in the previous subsection, the calculation is rather involved if one considers the general case including sources and fluxes. Let us therefore note that there is an alternative way to obtain our result, which only uses the equations of motion instead of exploiting the scaling symmetries. This second derivation may serve as a double-check of our results and is detailed in appendix A. In the following, we will continue to discuss the first method, using the scaling symmetries. The reader who is less interested in the technical details of the derivation may also jump directly to (3.33) and the subsequent discussion, where we present our result for $\Lambda$.

Let $\tau$ denote the scaling parameter, where $\tau$ equals $s$ if we consider the dilaton scaling (3.1) and $t$ in case of the mass scaling (3.4). Moreover, we will use primes to denote the $\tau$-transformed fields and the corresponding $\tau$-transformed action. Thus, if $\tau=s$, we have, for example, $g_{M N}^{\prime}=\sqrt{s} g_{M N}$, and if $\tau=t$, we have $g_{M N}^{\prime}=t^{-2} g_{M N}$. According to (3.3) and (3.6), the action (2.1) then scales as

$$
S^{\prime}=S_{\mathrm{bulk}}^{\prime}+S_{\mathrm{loc}}^{\prime}=\tau^{k} S_{\mathrm{bulk}}+\sum_{p} \tau^{l_{p}} S_{\mathrm{loc}}^{(p)}
$$

\footnotetext{
${ }^{6} \mathrm{~A}$ subtlety occurs for $F_{5}$, which is self-dual, and $F_{4}$, which can have both internal and spacetime-filling components in compactifications to 4 dimensions. In these cases, only the internal components $F_{4}^{\text {int }}, F_{5}^{\text {int }}$ can have a source term in the Bianchi identity. We therefore express those in terms of their duals $F_{6}^{\text {ext }}$, $F_{5}^{\text {ext }}$, which can in turn be written in terms of (3.13).
} 
where $k=2, l_{p}=1$ for $\tau=s$ and $k=-8, l_{p}=-p-1$ for $\tau=t$. Taking the $\tau$ derivative and evaluating the equation at $\tau=1$, we find

$$
\left.\frac{\mathrm{d} S_{\text {bulk }}^{\prime}}{\mathrm{d} \tau}\right|_{\tau=1}+\left.\frac{\mathrm{d} S_{\text {loc }}^{\prime}}{\mathrm{d} \tau}\right|_{\tau=1}=k S_{\text {bulk }}+\sum_{p} l_{p} S_{\text {loc }}^{(p)} .
$$

We now proceed as in the simple example discussed in section 3.2: we first evaluate the terms on the left-hand side of the equation and integrate by parts to express them in terms of a functional derivative of the action with respect to the fields. We then substitute the equations of motion to simplify the expressions.

The first term on the left-hand side of (3.15) yields ${ }^{7}$

$$
\left.\frac{\mathrm{d} S_{\text {bulk }}^{\prime}}{\mathrm{d} \tau}\right|_{\tau=1}=\left.\int\left[\frac{\delta S_{\text {bulk }}}{\delta g_{M N}} \frac{\mathrm{d} g_{M N}^{\prime}}{\mathrm{d} \tau}+\frac{\delta S_{\text {bulk }}}{\delta \phi} \frac{\mathrm{d} \phi^{\prime}}{\mathrm{d} \tau}+\frac{\delta S_{\text {NSNS }}}{\delta H} \wedge \frac{\mathrm{d} H^{\prime}}{\mathrm{d} \tau}+\left\langle\frac{\delta S_{\mathrm{RR}}}{\delta F} \wedge \frac{\mathrm{d} F^{\prime}}{\mathrm{d} \tau}\right\rangle_{10}\right]\right|_{\tau=1},
$$

where we have implicitly used partial integration to write the first two terms in the integrand as functional derivatives of $S_{\text {bulk }}$ with respect to the metric and the dilaton. These functional derivatives are equivalent to the variation of the bulk action, which will later allow us to use the equations of motion to simplify the expression. Similarly, we should also rewrite the remaining two terms in above equation as variations with respect to the NSNS and RR potentials. This is more involved since $H$ and $F$ may contain flux (cf. (3.10) and (3.13)), and so we will consider these terms separately later. Let us at first evaluate the $\mathrm{d} S_{\text {loc }}^{\prime} / \mathrm{d} \tau$ term in (3.15),

$$
\begin{aligned}
\left.\frac{\mathrm{d} S_{\mathrm{loc}}^{\prime}}{\mathrm{d} \tau}\right|_{\tau=1} & =\left.\int\left[\frac{\delta S_{\mathrm{loc}}}{\delta g_{M N}} \frac{\mathrm{d} g_{M N}^{\prime}}{\mathrm{d} \tau}+\frac{\delta S_{\mathrm{loc}}}{\delta \phi} \frac{\mathrm{d} \phi^{\prime}}{\mathrm{d} \tau}+\left\langle\frac{\delta S_{\mathrm{loc}}}{\delta C} \wedge \frac{\mathrm{d} C^{\prime}}{\mathrm{d} \tau}\right\rangle_{10}+\frac{\delta S_{\mathrm{loc}}}{\delta B} \wedge \frac{\mathrm{d} B^{\prime}}{\mathrm{d} \tau}\right]\right|_{\tau=1} \\
& =\left.\int\left[\frac{\delta S_{\mathrm{loc}}}{\delta g_{M N}} \frac{\mathrm{d} g_{M N}^{\prime}}{\mathrm{d} \tau}+\frac{\delta S_{\mathrm{loc}}}{\delta \phi} \frac{\mathrm{d} \phi^{\prime}}{\mathrm{d} \tau}\right]\right|_{\tau=1}+\left.\sum_{p} \frac{\mathrm{d} S_{\mathrm{CS}}^{(p)}}{\mathrm{d} \tau}\right|_{\tau=1}
\end{aligned}
$$

Since $S_{\text {loc }}$ does not depend on any field derivatives but only on the fields themselves, we did not have to integrate by parts here. We can now combine (3.16) and (3.17) and use the equations of motion $\delta S / \delta g_{M N}=\delta S / \delta \phi=0$ to obtain

$$
\left.\frac{\mathrm{d} S_{\text {bulk }}^{\prime}}{\mathrm{d} \tau}\right|_{\tau=1}+\left.\frac{\mathrm{d} S_{\mathrm{loc}}^{\prime}}{\mathrm{d} \tau}\right|_{\tau=1}=\left.\int\left[\frac{\delta S_{\mathrm{NSNS}}}{\delta H} \wedge \frac{\mathrm{d} H^{\prime}}{\mathrm{d} \tau}+\left\langle\frac{\delta S_{\mathrm{RR}}}{\delta F} \wedge \frac{\mathrm{d} F^{\prime}}{\mathrm{d} \tau}\right\rangle_{10}\right]\right|_{\tau=1}+\left.\sum_{p} \frac{\mathrm{d} S_{\mathrm{CS}}^{\prime(p)}}{\mathrm{d} \tau}\right|_{\tau=1} .
$$

The two terms involving $\delta H$ and $\delta F$ are evaluated as follows. Substituting (3.10) into the $\delta S_{\mathrm{NSNS}} / \delta H$ term in (3.18), we can integrate by parts to obtain

$$
\begin{aligned}
\left.\int \frac{\delta S_{\mathrm{NSNS}}}{\delta H} \wedge \frac{\mathrm{d} H^{\prime}}{\mathrm{d} \tau}\right|_{\tau=1} & =\left.\int\left[\mathrm{d} \frac{\delta S_{\mathrm{NSNS}}}{\delta H} \wedge \frac{\mathrm{d} B^{\prime}}{\mathrm{d} \tau}+\frac{\delta S_{\mathrm{NSNS}}}{\delta H} \wedge \frac{\mathrm{d} H^{\prime b}}{\mathrm{~d} \tau}\right]\right|_{\tau=1} \\
& =\left.\int\left[\frac{\delta S_{\mathrm{NSNS}}}{\delta B} \wedge \frac{\mathrm{d} B^{\prime}}{\mathrm{d} \tau}+\frac{\delta S_{\mathrm{NSNS}}}{\delta H} \wedge \frac{\mathrm{d} H^{\prime b}}{\mathrm{~d} \tau}\right]\right|_{\tau=1} .
\end{aligned}
$$

\footnotetext{
${ }^{7}$ We define functional derivatives with respect to form fields, $A$, such that $\delta S=\int \frac{\delta S}{\delta A} \wedge \delta A$.
} 
The $\delta S_{\mathrm{RR}} / \delta F$ term in (3.18) can be computed in a similar fashion but is more complicated due to the subtleties explained in section 3.2. We first use (3.11) and write

$$
\left.\int\left\langle\frac{\delta S_{\mathrm{RR}}}{\delta F} \wedge \frac{\mathrm{d} F^{\prime}}{\mathrm{d} \tau}\right\rangle_{10}\right|_{\tau=1}=\left.\int\left\langle\frac{\delta S_{\mathrm{RR}}}{\delta F^{\text {ext }}} \wedge \frac{\mathrm{d} F^{\prime \text { ext }}}{\mathrm{d} \tau}+\frac{\delta S_{\mathrm{RR}}}{\delta F^{\text {int }}} \wedge \frac{\mathrm{d} F^{\text {int }}}{\mathrm{d} \tau}\right\rangle_{10}\right|_{\tau=1} .
$$

We now have to replace all RR field strengths $F_{n}^{\text {int }}$ by their dual field strengths $F_{10-n}^{\text {ext }}$ in order to be able to write them in terms of the globally defined gauge potentials $C^{\text {ext }}$ using (3.13), which in turn will allow us to integrate by parts in (3.20). Using the duality relations (2.4) as well as the scalings (3.1) and (3.4), we find for the two cases $\tau=s$ and $\tau=t$ :

$$
\begin{aligned}
\left.\int\left\langle\frac{\delta S_{\mathrm{RR}}}{\delta F} \wedge \frac{\mathrm{d} F^{\prime}}{\mathrm{d} s}\right\rangle_{10}\right|_{s=1} & =\sum_{n} \int\left(\frac{\delta S_{\mathrm{RR}}}{\delta F_{n}^{\text {ext }}} \wedge F_{n}^{\mathrm{ext}}+\frac{\delta S_{\mathrm{RR}}}{\delta F_{n}^{\mathrm{int}}} \wedge F_{n}^{\mathrm{int}}\right) \\
& =\sum_{n} \int\left(\frac{\delta S_{\mathrm{RR}}}{\delta F_{n}^{\text {ext }}} \wedge F_{n}^{\mathrm{ext}}-\frac{\delta S_{\mathrm{RR}}}{\delta F_{10-n}^{\text {ext }}} \wedge F_{10-n}^{\mathrm{ext}}\right) \\
& =0 \\
\left.\int\left\langle\frac{\delta S_{\mathrm{RR}}}{\delta F} \wedge \frac{\mathrm{d} F^{\prime}}{\mathrm{d} t}\right\rangle_{10}\right|_{t=1} & =\sum_{n}(1-n) \int\left(\frac{\delta S_{\mathrm{RR}}}{\delta F_{n}^{\text {ext }}} \wedge F_{n}^{\mathrm{ext}}+\frac{\delta S_{\mathrm{RR}}}{\delta F_{n}^{\mathrm{int}}} \wedge F_{n}^{\mathrm{int}}\right) \\
& =\sum_{n}(1-n) \int\left(\frac{\delta S_{\mathrm{RR}}}{\delta F_{n}^{\text {ext }}} \wedge F_{n}^{\mathrm{ext}}-\frac{\delta S_{\mathrm{RR}}}{\delta F_{10-n}^{\mathrm{ext}}} \wedge F_{10-n}^{\mathrm{ext}}\right) \\
& =\sum_{n}(10-2 n) \int \frac{\delta S_{\mathrm{RR}}}{\delta F_{n}^{\text {ext }}} \wedge F_{n}^{\mathrm{ext}} .
\end{aligned}
$$

These two expressions can now be rewritten in a way that will become convenient further below. In order to do so, we again exploit the scalings (3.1) and (3.4) and make use of the identity $\delta S_{\mathrm{RR}} / \delta F_{n}^{\text {ext }} \wedge F_{n}^{\text {ext }}=-\frac{1}{2} \star_{10} \mathrm{e}^{(5-n) \phi / 2}\left|F_{n}^{\text {ext }}\right|^{2}$, which can be derived from (2.2). We thus find

$$
\begin{aligned}
\left.\int\left\langle\frac{\delta S_{\mathrm{RR}}}{\delta F} \wedge \frac{\mathrm{d} F^{\prime}}{\mathrm{d} \tau}\right\rangle_{10}\right|_{\tau=1}= & \left.2 \int\left\langle\frac{\delta S_{\mathrm{RR}}}{\delta F^{\mathrm{ext}}} \wedge \frac{\mathrm{d} F^{\prime \text { ext }}}{\mathrm{d} \tau}\right\rangle_{10}\right|_{\tau=1}-2 k \int\left\langle\frac{\delta S_{\mathrm{RR}}}{\delta F^{\text {ext }}} \wedge F^{\text {ext }}\right\rangle_{10} \\
& -\frac{k}{2} \sum_{n} \int \star_{10} \mathrm{e}^{\frac{5-n}{2} \phi}\left|F_{n}^{\text {ext }}\right|^{2}
\end{aligned}
$$

where $k=2$ for $\tau=s$ and $k=-8$ for $\tau=t$ as in (3.14).

We now integrate by parts on the right-hand side of equation (3.23). Taking into account (3.10) and (3.13), this yields ${ }^{8}$

$$
\left.\int\left\langle\frac{\delta S_{\mathrm{RR}}}{\delta F^{\mathrm{ext}}} \wedge \frac{\mathrm{d} F^{\text {ext }}}{\mathrm{d} \tau}\right\rangle_{10}\right|_{\tau=1}
$$

\footnotetext{
${ }^{8}$ The factor $\frac{1}{2}$ that appears when rewriting $\delta S_{\mathrm{RR}} / \delta C^{\mathrm{ext}}$ in terms of $\delta S / \delta C^{\text {ext }}$ and $\delta S_{\text {loc }} / \delta C^{\text {ext }}$ is related to a subtlety regarding the variation of the CS action of the RR fields. One only obtains the correct equations of motion if one takes the coupling of the RR fields to the sources as being half the coupling that one would get from the "naive" variation of the action. One can think of this as being due to the fact that one half of $\sum_{p} S_{\mathrm{CS}}^{(p)}$ represents an electric coupling of the RR fields to the sources, whereas the other half is due to a magnetic coupling of the dual RR fields to the sources. This subtlety is known in the literature and has, for example, been discussed in footnote 6 of [10].
} 


$$
\begin{aligned}
= & \left.\int\left\langle\frac{\delta S_{\mathrm{RR}}}{\delta F^{\text {ext }}} \wedge\left(\mathrm{d}_{-H} \frac{\mathrm{d} C^{\prime \text { ext }}}{\mathrm{d} \tau}+\mathrm{e}^{B} \wedge \frac{\mathrm{d} F^{\prime b}}{\mathrm{~d} \tau}-\frac{\mathrm{d}\left(\mathrm{d} B^{\prime}+H^{\prime b}\right)}{\mathrm{d} \tau} \wedge C^{\text {ext }}+\frac{\mathrm{d} B^{\prime}}{\mathrm{d} \tau} \wedge \mathrm{e}^{B} \wedge F^{b}\right)\right\rangle_{10}\right|_{\tau=1} \\
= & \left.\int\left\langle\frac{\delta S_{\mathrm{RR}}}{\delta C^{\text {ext }}} \wedge \frac{\mathrm{d} C^{\prime \text { ext }}}{\mathrm{d} \tau}+\frac{\delta S_{\mathrm{RR}}}{\delta F^{\text {ext }}} \wedge\left(\mathrm{e}^{B} \wedge \frac{\mathrm{d} F^{\prime b}}{\mathrm{~d} \tau}+\frac{\delta F^{\text {ext }}}{\delta B} \wedge \frac{\mathrm{d} B^{\prime}}{\mathrm{d} \tau}+\frac{\delta F^{\text {ext }}}{\delta H} \wedge \frac{\mathrm{d} H^{\prime b}}{\mathrm{~d} \tau}\right)\right\rangle_{10}\right|_{\tau=1} \\
= & \int\left\langle\left(\frac{\delta S}{\delta C^{\text {ext }}}-\frac{1}{2} \frac{\delta S_{\text {loc }}}{\delta C^{\text {ext }}}\right) \wedge \frac{\mathrm{d} C^{\prime \text { ext }}}{\mathrm{d} \tau}+\frac{1}{2} \frac{\delta S_{\mathrm{RR}}}{\delta B} \wedge \frac{\mathrm{d} B^{\prime}}{\mathrm{d} \tau}\right. \\
& \left.+\frac{\delta S_{\mathrm{RR}}}{\delta F^{\text {ext }}} \wedge\left(\mathrm{e}^{B} \wedge \frac{\mathrm{d} F^{\prime b}}{\mathrm{~d} \tau}+\frac{\delta F^{\text {ext }}}{\delta H} \wedge \frac{\mathrm{d} H^{\prime b}}{\mathrm{~d} \tau}\right)\right\rangle\left._{10}\right|_{\tau=1} \\
= & \left\langle\left(\frac{\delta S}{\delta C^{\text {ext }}}-\frac{1}{2} \frac{\delta S_{\mathrm{loc}}}{\delta C^{\text {ext }}}\right) \wedge \frac{\mathrm{d} C^{\prime \text { ext }}}{\mathrm{d} \tau}+\frac{1}{2}\left(\frac{\delta S}{\delta B}-\frac{\delta S_{\mathrm{NSNS}}}{\delta B}-\frac{\delta S_{\mathrm{loc}}}{\delta B}\right) \wedge \frac{\mathrm{d} B^{\prime}}{\mathrm{d} \tau}\right. \\
& \left.+\frac{\delta S_{\mathrm{RR}}}{\delta F^{\text {ext }}} \wedge\left(\mathrm{e}^{B} \wedge \frac{\mathrm{d} F^{\prime b}}{\mathrm{~d} \tau}+\frac{\delta F^{\text {ext }}}{\delta H} \wedge \frac{\mathrm{d} H^{\prime b}}{\mathrm{~d} \tau}\right)\right\rangle\left._{10}\right|_{\tau=1},
\end{aligned}
$$

where we also used

$$
2\left\langle\frac{\delta S_{\mathrm{RR}}}{\delta F^{\mathrm{ext}}} \wedge \frac{\delta F^{\mathrm{ext}}}{\delta B}\right\rangle_{8}=\left\langle F^{\mathrm{ext}} \wedge \sigma\left(F^{\mathrm{int}}\right)\right\rangle_{8}-\frac{\delta S_{\mathrm{loc}}}{\delta B}=\frac{\delta S}{\delta B}-\frac{\delta S_{\mathrm{NSNS}}}{\delta B}-\frac{\delta S_{\mathrm{loc}}}{\delta B}=\frac{\delta S_{\mathrm{RR}}}{\delta B},
$$

which can be derived using (2.2), (2.16), (3.10) and (3.13). With the equations of motion, $\delta S / \delta C^{\text {ext }}=\delta S / \delta B=0$, one finally obtains

$$
\begin{aligned}
\left.\int\left\langle\frac{\delta S_{\mathrm{RR}}}{\delta F^{\mathrm{ext}}} \wedge \frac{\mathrm{d} F^{\prime \text { ext }}}{\mathrm{d} \tau}\right\rangle_{10}\right|_{\tau=1}= & -\left.\frac{1}{2} \sum_{p} \frac{\mathrm{d} S_{\mathrm{CS}}^{\prime(p)}}{\mathrm{d} \tau}\right|_{\tau=1}-\left.\frac{1}{2} \int \frac{\delta S_{\mathrm{NSNS}}}{\delta B} \wedge \frac{\mathrm{d} B^{\prime}}{\mathrm{d} \tau}\right|_{\tau=1} \\
& +\left.\int\left\langle\frac{\delta S_{\mathrm{RR}}}{\delta F^{\mathrm{ext}}} \wedge\left(\mathrm{e}^{B} \wedge \frac{\mathrm{d} F^{\prime b}}{\mathrm{~d} \tau}+\frac{\delta F^{\mathrm{ext}}}{\delta H} \wedge \frac{\mathrm{d} H^{\prime b}}{\mathrm{~d} \tau}\right)\right\rangle_{10}\right|_{\tau=1}
\end{aligned}
$$

Evaluating this for $\tau=s$ using (3.1) then also implies

$$
\int\left\langle\frac{\delta S_{\mathrm{RR}}}{\delta F^{\mathrm{ext}}} \wedge F^{\mathrm{ext}}\right\rangle_{10}=-\frac{1}{2} \sum_{p} S_{\mathrm{CS}}^{(p)}+\int\left\langle\frac{\delta S_{\mathrm{RR}}}{\delta F^{\mathrm{ext}}} \wedge \mathrm{e}^{B} \wedge F^{b}\right\rangle_{10} .
$$

Substituting (3.26) and (3.27) into (3.23) then leads to

$$
\begin{aligned}
\left.\int\left\langle\frac{\delta S_{\mathrm{RR}}}{\delta F} \wedge \frac{\mathrm{d} F^{\prime}}{\mathrm{d} \tau}\right\rangle_{10}\right|_{\tau=1}= & -\left.\sum_{p} \frac{\mathrm{d} S_{\mathrm{CS}}^{(p)}}{\mathrm{d} \tau}\right|_{\tau=1}+k \sum_{p} S_{\mathrm{CS}}^{(p)}-\left.\int \frac{\delta S_{\mathrm{NSNS}}}{\delta B} \wedge \frac{\mathrm{d} B^{\prime}}{\mathrm{d} \tau}\right|_{\tau=1} \\
& +\left.2 \int\left\langle\frac{\delta S_{\mathrm{RR}}}{\delta F^{\mathrm{ext}}} \wedge\left(\mathrm{e}^{B} \wedge \frac{\mathrm{d} F^{\prime b}}{\mathrm{~d} \tau}-k \mathrm{e}^{B} \wedge F^{b}+\frac{\delta F^{\mathrm{ext}}}{\delta H} \wedge \frac{\mathrm{d} H^{\prime b}}{\mathrm{~d} \tau}\right)\right\rangle_{10}\right|_{\tau=1} \\
& -\frac{k}{2} \sum_{n} \int \star_{10} \mathrm{e}^{\frac{5-n}{2} \phi}\left|F_{n}^{\mathrm{ext}}\right|^{2}
\end{aligned}
$$

Putting everything together, we now use (3.28) together with (3.19) in (3.18) to arrive at

$$
\left[\frac{\mathrm{d} S_{\text {bulk }}^{\prime}}{\mathrm{d} \tau}+\frac{\mathrm{d} S_{\text {loc }}^{\prime}}{\mathrm{d} \tau}\right]_{\tau=1}=k \sum_{p} S_{\mathrm{CS}}^{(p)}-\frac{k}{2} \sum_{n} \int \star_{10} \mathrm{e}^{\frac{5-n}{2} \phi}\left|F_{n}^{\text {ext }}\right|^{2}+\left.\int \frac{\delta S_{\mathrm{NSNS}}}{\delta H} \wedge \frac{\mathrm{d} H^{\prime b}}{\mathrm{~d} \tau}\right|_{\tau=1}
$$




$$
+\left.2 \int\left\langle\frac{\delta S_{\mathrm{RR}}}{\delta F^{\mathrm{ext}}} \wedge\left(\mathrm{e}^{B} \wedge \frac{\mathrm{d} F^{\prime b}}{\mathrm{~d} \tau}-k \mathrm{e}^{B} \wedge F^{b}+\frac{\delta F^{\mathrm{ext}}}{\delta H} \wedge \frac{\mathrm{d} H^{\prime b}}{\mathrm{~d} \tau}\right)\right\rangle_{10}\right|_{\tau=1}
$$

Using (3.15) and the two scaling symmetries (3.1) and (3.4) and evaluating the functional derivatives then leads to the two equations

$$
\begin{aligned}
2 S_{\text {bulk }}+S_{\text {loc }}= & 2 \sum_{p} S_{\mathrm{CS}}^{(p)}-\sum_{n} \int \star_{10} \mathrm{e}^{\frac{5-n}{2} \phi}\left|F_{n}^{\text {ext }}\right|^{2} \\
& -\sum_{n} \int F_{n}^{b} \wedge\left\langle\mathrm{e}^{B} \wedge \sigma\left(F^{\mathrm{int}}\right)\right\rangle_{10-n}, \\
-8 S_{\text {bulk }}-\sum_{p}(p+1) S_{\mathrm{loc}}^{(p)}= & -8 \sum_{p} S_{\mathrm{CS}}^{(p)}+4 \sum_{n} \int \star_{10} \mathrm{e}^{\frac{5-n}{2} \phi}\left|F_{n}^{\mathrm{ext}}\right|^{2} \\
& +\sum_{n}(9-n) \int F_{n}^{b} \wedge\left\langle\mathrm{e}^{B} \wedge \sigma\left(F^{\mathrm{int}}\right)\right\rangle_{10-n} \\
& -2 \int H^{b} \wedge\left(\mathrm{e}^{-\phi} \star_{10} H-\left\langle\sigma\left(F^{\mathrm{int}}\right) \wedge C^{\mathrm{ext}}\right\rangle_{7}\right),
\end{aligned}
$$

where $\sigma$ is the operator defined in (2.5). We can now linearly combine (3.30) and (3.31) introducing a free parameter $c$ and rearrange the source terms using $S=S_{\text {bulk }}+S_{\text {loc }}$ and $S_{\mathrm{loc}}^{(p)}=S_{\mathrm{DBI}}^{(p)}+S_{\mathrm{CS}}^{(p)}$, which yields

$$
\begin{aligned}
2 S-2 \sum_{p} S_{\mathrm{CS}}^{(p)}+\sum_{n} \int \star_{10} \mathrm{e}^{\frac{5-n}{2} \phi}\left|F_{n}^{\mathrm{ext}}\right|^{2}= & \sum_{p}\left(1+\frac{p-3}{2} c\right)\left[S_{\mathrm{DBI}}^{(p)}+S_{\mathrm{CS}}^{(p)}\right] \\
& -\sum_{n}\left(1+\frac{n-5}{2} c\right) \int F_{n}^{b} \wedge\left\langle\mathrm{e}^{B} \wedge \sigma\left(F^{\mathrm{int}}\right)\right\rangle_{10-n} \\
& -c \int H^{b} \wedge\left(\mathrm{e}^{-\phi} \star_{10} H-\left\langle\sigma\left(F^{\mathrm{int}}\right) \wedge C^{\mathrm{ext}}\right\rangle_{7}\right) .
\end{aligned}
$$

Substituting this into the integrated Einstein equation (2.12) and collecting all contributions from background fluxes into a single term $\mathcal{F}(c)$, we find the result

$$
\frac{8 v \mathcal{V}}{d-2} \Lambda=\sum_{p}\left(1+\frac{p-3}{2} c\right)\left[S_{\mathrm{DBI}}^{(p)}+S_{\mathrm{CS}}^{(p)}\right]+\int \mathcal{F}(c)
$$

with the volume factors $v$ and $\mathcal{V}$ defined as in (2.13). Note that all terms on the right-hand side of (3.33) contain an implicit factor of the external "volume" $v$ such that it cancels out in the equation, and $\Lambda$ does not depend on it. The flux term $\mathcal{F}(c)$ takes the form

$$
\begin{aligned}
\mathcal{F}(c)= & -\sum_{n \geq d}\left(1+\frac{n-5}{2} c\right) F_{n}^{b} \wedge\left\langle\mathrm{e}^{B} \wedge \sigma\left(F^{\mathrm{int}}\right)\right\rangle_{10-n} \\
& -c H^{b} \wedge\left(\mathrm{e}^{-\phi} \star_{10} H-\left\langle\sigma\left(F^{\mathrm{int}}\right) \wedge C^{\mathrm{ext}}\right\rangle_{7}\right),
\end{aligned}
$$


where the summation range is determined by the fact that the background fluxes $F_{n}^{b}$ are spacetime-filling by definition and must therefore be of rank $d$ or higher (cf. the discussion in section 3.2).

As stated earlier, the contribution of the flux term $\mathcal{F}(c)$ can often be gauged away in (3.33) by choosing an appropriate numerical value for the free parameter $c$. Up to an overall volume factor $\mathcal{V}$ (whose sign is known to be positive), $\Lambda$ is then completely determined by the on-shell actions of the localized sources that appear in the corresponding solution. If only one of the fluxes in (3.34) is non-zero, it is straightforward to see that $\mathcal{F}(c)$ can be set to zero, since then one can simply choose $c$ such that the $c$-dependent prefactor of the corresponding term vanishes in (3.34). ${ }^{9}$ For a compactification with non-zero $H^{b}$, for example, one would choose $c=0$, and, for a compactification with non-zero $F_{7}^{b}$, one would choose $c=-1$.

Even if the NSNS flux $H^{b}$ and one of the RR fluxes (other than $F_{5}^{b}$ ) are both non-zero, it is still often possible to find a $c$ such that $\mathcal{F}(c)$ vanishes. The reason is that the term multiplying $H^{b}$ in (3.34) is proportional to

$$
\frac{\delta S_{\mathrm{NSNS}}}{\delta H}+2\left\langle\frac{\delta S_{\mathrm{RR}}}{\delta F^{\mathrm{ext}}} \wedge \frac{\delta F^{\mathrm{ext}}}{\delta H}\right\rangle_{7}=-\mathrm{e}^{-\phi} \star_{10} H+\left\langle\sigma\left(F^{\mathrm{int}}\right) \wedge C^{\mathrm{ext}}\right\rangle_{7} .
$$

If the $H$ equation of motion implies that $\mathrm{d}\left[\mathrm{e}^{-\phi} \star_{10} H-\left\langle\sigma\left(F^{\mathrm{int}}\right) \wedge C^{\mathrm{ext}}\right\rangle_{7}\right]=0$, which is the case in many interesting examples, then we can write

$$
-\mathrm{e}^{-\phi} \star_{10} H+\left\langle\sigma\left(F^{\mathrm{int}}\right) \wedge C^{\mathrm{ext}}\right\rangle_{7}=\omega_{7},
$$

where $\omega_{7}$ is a closed but not necessarily exact 7 -form. Note that only a possible non-exact part of $\omega_{7}$ can contribute to (3.33) since any exact part of $\omega_{7}$ would reduce to zero when inserted into (3.34) and integrated over. If a gauge transformation of the RR potentials can be employed to cancel $\omega_{7}$ in (3.36), the term multiplying $H^{b}$ in (3.34) vanishes for any $c$, and we can choose the value for $c$ such that also the RR flux term in (3.34) vanishes. Consider, for example, a compactification of type IIA supergravity with non-zero $H^{b}$ and $F_{0}$. The non-trivial background fluxes appearing in (3.34) are then $H^{b}$ and $F_{10}^{b}$,

$$
\mathcal{F}(c)=-\left(1+\frac{5}{2} c\right) F_{10}^{b} \wedge F_{0}-c H^{b} \wedge\left(\mathrm{e}^{-\phi} \star_{10} H-\left\langle\sigma\left(F^{\mathrm{int}}\right) \wedge C^{\mathrm{ext}}\right\rangle_{7}\right) .
$$

Assuming that $\mathrm{d}\left[\mathrm{e}^{-\phi} \star_{10} H-\left\langle\sigma\left(F^{\text {int }}\right) \wedge C^{\text {ext }}\right\rangle_{7}\right]=0$ by the $H$ equation of motion, (3.35) and (3.36) now imply that the term multiplying $H^{b}$ can be canceled by a gauge transformation $C_{7} \mapsto C_{7}-\omega_{7} / F_{0}$. This is a valid gauge transformation that leaves all RR field strengths unchanged. In the new gauge, we then have $\mathrm{e}^{-\phi} \star_{10} H-\left\langle\sigma\left(F^{\text {int }}\right) \wedge C^{\text {ext }}\right\rangle_{7}=0$ such that (3.37) reduces to $\mathcal{F}(c)=-(1+5 c / 2) F_{10}^{b} \wedge F_{0}$. We can therefore choose $c=-2 / 5$ so that $\mathcal{F}=0 .{ }^{10}$

\footnotetext{
${ }^{9} F_{5}$ flux is an exception, because it does not have a $c$-dependent prefactor in $\mathcal{F}(c)$ and can therefore not be gauged away in (3.33). This is the reason for the existence of the Freund-Rubin solutions of type IIB supergravity on $\mathrm{AdS}_{5} \times S^{5}[30]$.

${ }^{10}$ Note that, even though $\mathcal{F}(c)$ is not gauge invariant, one can convince oneself that the full expression for $\Lambda$ in (3.33) is gauge invariant.
} 
In presence of more than one type of RR flux, this reasoning does not work anymore, since it is then not possible to choose an appropriate $c$ such that each term in $\mathcal{F}(c)$ is set to zero individually. We may still be able to find a $c=c_{0}$ that solves the equation $\int \mathcal{F}\left(c_{0}\right)=0$ such that $\int \mathcal{F}\left(c_{0}\right)$ vanishes as a whole, but the numerical value of $c_{0}$ then depends on the bulk fields that appear in (3.34). This will in general not be useful, since it just has the effect of trading the explicit dependence of $\Lambda$ on the bulk dynamics for an implicit dependence hidden in the value of $c_{0}$. We will explain this in more detail in section 4 where we discuss several examples for string compactifications in which $\mathcal{F}(c)$ can be set to zero and one counterexample in which it cannot be set to zero.

\subsection{Validity of the supergravity approximation}

Before we proceed with applying the above results to some explicit examples, a comment on their regime of validity is in order. In the vicinity of localized sources, field derivatives and the string coupling often blow up such that $\alpha^{\prime}$ and loop corrections can become large, making the reliability of the supergravity approximation questionable. Given that the right-hand side of (3.33) is evaluated directly at the positions of the sources, one might therefore wonder about the self-consistency of our expression for $\Lambda$.

In order to clarify the meaning of our result, it is important to recall that (3.33) has been derived by using the two-derivative supergravity action $(2.1),(2.2),(2.6),(2.7)$. Within this theory, (3.33) is an exact expression that can serve as well as any other method for calculating the cosmological constant in the supergravity approximation. The only question now is what happens to (3.33) if one takes into account the various types of stringy corrections, because these may significantly affect the strong field region at the sources.

The answer to this question depends on how (3.33) is used. If one reads it as an expression that calculates the cosmological constant in terms of the near-source behavior, one has to use the near-source behavior in the supergravity approximation and then gets the cosmological constant in the supergravity approximation. Let us, for simplicity, focus on the case with only one type of sources present in the compactification. We can then schematically write $\Lambda^{\text {class }}=\kappa S_{\text {loc }}^{\text {class }}$, where the superscript ${ }^{\text {class }}$ denotes the values in the supergravity appoximation, and $\kappa$ is some constant. If classical supergravity provides a good approximation for the lower-dimensional effective theory, e.g. in the usual regime of large volume and small string coupling, the full cosmological constant, $\Lambda^{\text {full }}$, is well-approximated by the lowest order expression, $\Lambda^{\text {full }} \approx \Lambda^{\text {class }}$, and one therefore also has $\Lambda^{\text {full }} \approx \kappa S_{\text {loc }}^{\text {class }}$. Note that this is true even when $S_{\text {loc }}^{\text {class }}$ is not a good approximation to $S_{\text {loc }}^{\text {full }}$. This is the way we will use (3.33) in section 4 .

In section 5, on the other hand, we also use (3.33) backwards, i.e. we extract information on the near-brane behavior in a setup where $\Lambda$ is known. Here it is important to stress that this will only give us information on $S_{\text {loc }}^{\text {class }}$, i.e. on the near brane behavior in the supergravity approximation. In particular, the singularity in the $H$ and $F_{3}$ energy density we find is a priori only a feature of the supergravity approximation, and our result just confirms the singularity exactly like other people have seen the singularity in the supergravity approximation [14-17, 20]. Whether the singularity gets resolved by stringy effects can not be inferred from our argument and is beyond the scope of our work. The useful advantage 
of our method is that it shows that this singularity survives the full supergravity analysis and is not an artifact of the partial smearing or a linearization around the BPS background.

\section{Examples}

In this section, we discuss different solutions of type IIA and IIB supergravity that have appeared in the literature and show how (3.33) can be evaluated in our framework to obtain an explicit expression for the cosmological constant.

\subsection{The GKP solutions}

Here we consider warped compactifications of type IIB supergravity to 4-dimensional Minkowski space with $H$ flux and $F_{3}$ flux and the necessary sources for tadpole cancelation along the lines of [10] (GKP) and related work [31-34]. For simplicity, we specialize to models involving only O3-planes as sources. In [10], the authors also discussed models with D7-branes and O7-planes along with their F-theory description. The discussion of models with 7-branes in our framework is analogous albeit more lengthy.

Following [10], we find that the non-vanishing fields must satisfy

$$
F_{3}=-\mathrm{e}^{-\phi} \star_{6} H, \quad F_{5}=-\left(1+\star_{10}\right) \mathrm{e}^{-4 A} \star_{6} \mathrm{~d} \alpha, \quad C_{4}^{\text {ext }}=\tilde{\star}_{4}(\alpha+a), \quad \alpha=\mathrm{e}^{4 A},
$$

where the warp factor $A$ and the dilaton $\phi$ are functions on the compact space, and $a$ is an integration constant corresponding to a gauge transformation. Also note that $F_{5}=\star_{10} F_{5}=F_{5}^{\text {int }}+F_{5}^{\text {ext }}$ with $F_{5}^{\text {ext }}=\mathrm{d} C_{4}^{\text {ext }}$. The topologically non-trivial fluxes canceling the O3-tadpoles are $F_{3}$ flux and $H$ flux, so that the relevant fluxes appearing in the definition of $\mathcal{F}(c)$, given by (3.34), are

$$
H^{b} \text { and } F_{7}^{b} \text {, }
$$

whereas all other terms in (3.34) vanish. Thus (3.34) reduces to

$$
\mathcal{F}(c)=-c H^{b} \wedge\left[\mathrm{e}^{-\phi} \star_{10} H+F_{3} \wedge C_{4}^{\mathrm{ext}}\right]+(1+c) F_{7}^{b} \wedge F_{3}
$$

Using (4.1), we find that the first term can be put to zero by gauge fixing $a=0 .{ }^{11}$ Furthermore, $F_{3}$ and $H$ are related by a special condition which is given in (4.1). This condition can be shown to saturate a BPS-like bound and is equivalent to the ISD condition of the complex three-form field strength in the notation of [10]. It follows from this condition that also the second term in (4.3) is zero, as can be checked:

$$
\begin{aligned}
\int F_{7}^{b} \wedge F_{3} & =\int\left(F_{7}-\mathrm{d} C_{6}^{\text {ext }}+H \wedge C_{4}^{\text {ext }}\right) \wedge F_{3} \\
& =\int\left(F_{7} \wedge F_{3}+\mathrm{e}^{\phi} \star_{6} F_{3} \wedge\left(\tilde{\star}_{4} \mathrm{e}^{4 A}\right) \wedge F_{3}\right)=0,
\end{aligned}
$$

\footnotetext{
${ }^{11}$ Note that, although $\mathcal{F}(c)$ is not gauge-invariant, the full expression for the cosmological constant $\Lambda$ is, since it contains a term $C_{4} \wedge \mu_{3} \delta_{6}$ which changes such that the total $a$-dependence of $\Lambda$ cancels out as it should.
} 
where in the last step we used that $F_{7}=-\mathrm{e}^{\phi} \star_{10} F_{3}=-\mathrm{e}^{\phi} \star_{6} F_{3} \wedge \tilde{\star}_{4} \mathrm{e}^{4 A}$. Thus $\mathcal{F}(c)$ reduces to zero for any choice of $c$. This is expected in this model, since also the contribution of localized source terms to $\Lambda$ is independent of $c$ for sources with $p=3$.

We therefore find that (3.33) yields

$$
\Lambda=\frac{1}{4 v \mathcal{V}}\left(S_{\mathrm{DBI}}^{(3)}+S_{\mathrm{CS}}^{(3)}\right) .
$$

Spelling out the contributions from the O3-planes and using (4.1) in (2.7), we arrive at

$$
\Lambda=\frac{1}{4 v \mathcal{V}} \mu_{3} \int\left(\tilde{\star}_{4} \mathrm{e}^{4 A}-C_{4}^{\mathrm{ext}}\right) \wedge \sigma\left(\delta_{6}\right)=\frac{1}{4 \mathcal{V}} N_{\mathrm{O} 3} \mu_{3}\left(\mathrm{e}^{4 A_{0}}-\alpha_{0}\right),
$$

where $A_{0}, \alpha_{0}$ denote the values of $A, \alpha$ at the position of the O3-plane(s) and $\mu_{3}>0$ is the absolute value of the $\mathrm{O} 3$ charge. Since $\alpha=\mathrm{e}^{4 A}$, the DBI and Chern-Simons parts of the source action cancel out such that

$$
\Lambda=0
$$

as expected.

\section{$4.2 \overline{\mathrm{D} 6}$-branes on $\mathrm{AdS}_{7} \times S^{3}$}

Let us now consider type IIA supergravity with $\overline{\mathrm{D} 6}$-branes on $\mathrm{AdS}_{7} \times S^{3}$, i.e. the setup studied in $[3,24,25] .{ }^{12}$ While a smeared solution can be constructed explicitly for this setup, it was argued in $[3,24]$ that in the supergravity approximation a solution with fully localized branes, if existent at all, necessarily yields a singularity in the energy density of the $H$ flux at the location of the $\overline{D 6}$-branes. As we will see below, it is rather straightforward to reproduce this result in our framework.

It was shown in [3] that the non-vanishing fields in this setup must satisfy the ansatz

$$
F_{0}=\text { const. }, \quad H=\alpha F_{0} \mathrm{e}^{\phi-7 A} \star_{3} 1, \quad F_{2}=\mathrm{e}^{-3 / 2 \phi-7 A} \star_{3} \mathrm{~d} \alpha, \quad C_{7}^{\text {ext }}=\tilde{\star}_{7}(\alpha+a),
$$

where the warp factor $A$, the dilaton $\phi$ and $\alpha$ are functions on the internal space, and $a$ is an integration constant related to a gauge freedom. The tadpole for the $\overline{\mathrm{D} 6}$-branes is canceled by a non-zero $H$ flux on the 3 -sphere and a non-zero Romans mass, i.e. $F_{0}$ "flux". The relevant fluxes appearing in $\mathcal{F}(c)$ are therefore

$$
H^{b} \text { and } F_{10}^{b} \text {, }
$$

and (3.34) reduces to

$$
\mathcal{F}(c)=-c H^{b} \wedge\left[\mathrm{e}^{-\phi} \star_{10} H-F_{0} \wedge C_{7}^{\mathrm{ext}}\right]-\left(1+\frac{5}{2} c\right) F_{10}^{b} \wedge F_{0}
$$

Using (4.8), one can see that the first term vanishes by a convenient gauge choice, $a=0$. We are then left with the second term which can be set to zero choosing $c=-\frac{2}{5}$.

\footnotetext{
${ }^{12}$ Note that, unlike in the scenario considered in [12], the anti-branes are here not added to uplift an existing AdS solution to dS, but to cancel the tadpole and guarantee the existence of an AdS solution in the first place.
} 
We can now substitute this into (3.33) to find

$$
\Lambda=\frac{1}{4 v \mathcal{V}}\left(S_{\mathrm{DBI}}^{(6)}+S_{\mathrm{CS}}^{(6)}\right)
$$

Spelling out the contributions of the $\overline{\mathrm{D} 6}$-branes and using (4.8) then yields

$$
\Lambda=\frac{1}{4 v \mathcal{V}} \mu_{6} \int\left(-\tilde{\star}_{7} \mathrm{e}^{3 / 4 \phi+7 A}-C_{7}^{\mathrm{ext}}\right) \wedge \sigma\left(\delta_{3}\right)=-\frac{1}{4 \mathcal{V}} N_{\overline{\mathrm{D} 6}} \mu_{6}\left(\mathrm{e}^{3 / 4 \phi_{0}+7 A_{0}}+\alpha_{0}\right),
$$

where $A_{0}, \alpha_{0}, \phi_{0}$ denote the values of $A, \alpha, \phi$ at the brane position and $\mu_{6}>0$ is the absolute value of the $\overline{\mathrm{D} 6}$ charge. Assuming that at leading order in the distance $r$ to the brane, the dilaton and the warp factor diverge as they would in flat space [35],

$$
\mathrm{e}^{2 A} \sim r^{1 / 8}, \quad \mathrm{e}^{\phi} \sim r^{3 / 4}
$$

it is straightforward to show that the first term in (4.12) (which comes from the DBI part of the brane action) is actually zero. That this assumption is correct was explicitly proven in the analysis carried out in [24].

The cosmological constant is therefore exclusively determined by $\alpha_{0}$ :

$$
\Lambda \sim-\mu_{6} \alpha_{0}
$$

Since $\Lambda$ is negative, it then follows that $\alpha$ has to be non-zero and positive at the source. Together with (4.13), this implies that near the source the energy density of the $H$ flux diverges like the inverse of the warp factor,

$$
\mathrm{e}^{-\phi}|H|^{2}=\alpha^{2} \mathrm{e}^{-14 A} \mathrm{e}^{\phi} F_{0}^{2} \sim \mathrm{e}^{-2 A} .
$$

This is consistent with the result found in $[3,24]$ by other methods, where it was also argued that finite $\alpha_{0}$ implies a singular energy density of the $H$ flux. As we will show in section 5, a similar argument holds for meta-stable de Sitter vacua that are obtained by placing $\overline{\mathrm{D} 3}$-branes on the Klebanov-Strassler throat embedded into a compact space. Under a few assumptions we will discuss in detail, one would find a singularity similar to the one observed in the $\overline{\mathrm{D} 6}$ model.

\subsection{SU(3)-structure Manifolds with O6-planes}

Here we discuss a particular model of compactifications of type IIA supergravity on SU(3)structure manifolds that was studied in [36], namely O6-planes on $\mathrm{dS}_{4} \times \mathrm{SU}(2) \times \mathrm{SU}(2)$ (see also [37] for more examples of this type). This setup allows (unstable) critical points with positive $\Lambda$.

According to [36], the form fields satisfy

$$
F_{0}=m, \quad F_{2}=m^{i} Y_{i}^{(2-)}, \quad H=p\left(Y_{1}^{(3-)}+Y_{2}^{(3-)}-Y_{3}^{(3-)}+Y_{4}^{(3-)}\right),
$$

where $Y_{i}^{(2-)}, Y_{i}^{(3-)}$ are certain 2-forms and 3-forms, respectively, and $m, m^{i}, p$ are constant coefficients that are not relevant for the following discussion. The tadpole generated by 
the O6-planes is canceled by non-zero $H$ and $F_{0}$ flux. However, while there is a non-trivial field strength $F_{2}$ (induced by the presence of the O6-planes), there is no topological $F_{2}$ flux, since it is not allowed by the cohomology of $\mathrm{SU}(2) \times \mathrm{SU}(2)$. For the same reason, $F_{8}^{b}=0$, and the non-zero background fluxes appearing in $\mathcal{F}(c)$ are

$$
H^{b} \text { and } F_{10}^{b}
$$

Considering (3.34) for this setup, we thus find

$$
\mathcal{F}(c)=-c H^{b} \wedge\left[\mathrm{e}^{-\phi} \star_{10} H-F_{0} \wedge C_{7}^{\mathrm{ext}}\right]-\left(1+\frac{5}{2} c\right) F_{10}^{b} \wedge F_{0} .
$$

As discussed in section 3.3, the $H$ equation of motion

$$
\mathrm{d}\left[\mathrm{e}^{-\phi} \star_{10} H-F_{0} \wedge C_{7}^{\mathrm{ext}}\right]=0
$$

implies that we can choose a gauge for $C_{7}^{\text {ext }}$ such that the first term on the right-hand side of (4.18) vanishes. The second term can be set to zero by choosing $c=-\frac{2}{5}$.

Evaluating (3.33), we therefore find that the cosmological constant is given by

$$
\Lambda=\frac{1}{10 v \mathcal{V}}\left(S_{\mathrm{DBI}}^{(6)}+S_{\mathrm{CS}}^{(6)}\right)=\frac{1}{10 v \mathcal{V}} \mu_{6} \int\left(\mathrm{e}^{3 / 4 \phi} \star_{4} 1 \wedge \star_{3} 1-C_{7}^{\mathrm{ext}}\right) \wedge \sigma\left(\delta_{3}\right),
$$

where the right hand side should be understood as a sum over the various O6-plane terms, and $\mu_{6}>0$ is the absolute value of the O6 charge. In [36], the setup was considered in the smeared limit, where the delta forms $\delta_{3}$ are replaced by volume forms of the space transverse to the corresponding sources. If a localized version of this solution exists, (4.20) would give a constraint on the possible field behavior at the O-planes.

\subsection{The DGKT solutions}

Finally, we look at type IIA supergravity compactified on $T^{6} / \mathbb{Z}_{3}^{2}$, which is an explicit example for the type IIA flux compactifications considered in $[38,39] .{ }^{13}$ In order to stabilize the moduli, the model requires the presence of NSNS flux as well as several RR fluxes of different ranks. As discussed in section 3.3, it is therefore a counterexample, where it is in general not possible to set the flux-dependent terms in (3.33) to zero and write $\Lambda$ as a sum of localized source terms only.

The NSNS and RR field strengths in this model are given by

$$
H^{b}=-p \beta_{0}, \quad F_{0}=m_{0}, \quad F_{2}=0, \quad F_{4}=F_{4}^{\text {int }}+F_{4}^{\text {ext }}=e_{i} \tilde{\omega}^{i}+\star_{4} e_{0},
$$

where $p, m_{0}, e_{0}, e_{i}$ are numbers, $\beta_{0}$ is an odd 3 -form and $\tilde{\omega}^{i}$ are even 4 -forms under the orientifold involution. ${ }^{14}$ The non-trivial fluxes appearing in (3.34) are thus

$$
H^{b}, \quad F_{10}^{b}, \quad F_{6}^{b} \quad \text { and } \quad F_{4}^{b}
$$

\footnotetext{
${ }^{13}$ As discussed in [39], the sources are smeared in order to obtain a solution. The discussion whether a corresponding localized solution exists or how it differs from the smeared solution [1-3, 39-42] does not concern us here. We only consider this model to give an example of a solution where many fluxes are turned on.

${ }^{14}$ Note that the spacetime-filling part of $F_{4}$, which is given by $F_{4}^{\text {ext }}$, is treated as internal $F_{6}$ in the conventions of [38].
} 
such that

$$
\begin{aligned}
\mathcal{F}(c)= & -c H^{b} \wedge\left[\mathrm{e}^{-\phi} \star_{10} H-F_{0} \wedge C_{7}^{\mathrm{ext}}\right]-\left(1+\frac{5}{2} c\right) F_{10}^{b} \wedge F_{0} \\
& -\left(1+\frac{1}{2} c\right) F_{6}^{b} \wedge F_{4}^{\mathrm{int}}+\left(1-\frac{1}{2} c\right) F_{4}^{b} \wedge F_{6}^{\mathrm{int}},
\end{aligned}
$$

where we used that the fluctuation $B$ is zero on-shell. The first term on the right-hand side can be made to vanish by choosing a gauge for the $C_{7}^{\text {ext }}$ field. Since the other terms do in general not vanish, however, we cannot choose $c$ such that all of them are set to zero simultaneously.

As pointed out in section 3.3, we can still solve the equation $\int \mathcal{F}(c)=0$ for some $c=c_{0}$ (unless its $c$-dependence coincidentally cancels out on-shell) and use it in (3.33) to arrive at an expression for $\Lambda$ which formally only depends on source terms,

$$
\Lambda=\frac{2+3 c_{0}}{8 v \mathcal{V}}\left(S_{\mathrm{DBI}}^{(6)}+S_{\mathrm{CS}}^{(6)}\right) .
$$

However, the resulting numerical value for $c_{0}$ then implicitly depends on the bulk fields appearing in $\mathcal{F}(c)$. It is therefore hard to approximate its numerical value or even its sign in compactification scenarios with more than one type of RR flux, unless the full solution is already known (as in the present example). This is contrary to the previous examples, where $c$ could be fixed to a known number such that, up to a volume factor, $\Lambda$ was completely determined by the boundary conditions of the fields in the near-source region.

\section{Singular $\overline{\mathrm{D} 3}$-branes in the Klebanov-Strassler throat}

In this section, we discuss to what extent our previous results can be applied to meta-stable de Sitter vacua in type IIB string theory obtained by placing $\overline{\mathrm{D} 3}$-branes at the tip of a warped throat geometry along the lines of [12]. We spell out and discuss the assumptions under which one can give a simple topological argument for a singularity in the energy density of $H$ and $F_{3}$ due to the brane backreaction.

\subsection{Ansatz}

Following [12], we consider type IIB no-scale Minkowski solutions obtained by embedding the Klebanov-Strassler solution [9] into a compact setting [10]. In order to stabilize the geometric moduli, we also include non-perturbative effects which may come from Euclidean D3-brane instantons or gaugino condensation. The resulting supersymmetric AdS vacuum is then uplifted to a meta-stable de Sitter vacuum by putting a small number of $\overline{\mathrm{D} 3}$-branes at the tip of the Klebanov-Strassler throat $[11,12]$.

In order to apply the results of section 3 to this scenario, we split the total cosmological constant into a part, $\Lambda^{\text {class }}$, which is due to the classical equations of motion and given by evaluating (3.33) at the solution, and the rest, $\Lambda^{\mathrm{np}}$, which contains all corrections from non-perturbative effects that are not captured by the classical computation, i.e., we write

$$
\Lambda=\Lambda^{\text {class }}+\Lambda^{\mathrm{np}} .
$$


Let us now discuss the explicit form of $\Lambda^{\text {class }}$ in the present setup. For simplicity, we will restrict ourselves to the case, where the no-scale solutions of [10] are realized in a model with O3-planes, and the non-perturbative effects come from Euclidean D3-brane instantons. In [10], also orientifold limits of F-theory compactifications involving D7-branes and O7-planes are discussed. We checked that it is also possible to study such models in our framework, but the discussion becomes more involved, since the presence of these sources induces a non-trivial $F_{1}$ field strength.

Our ansatz for the different fields thus reads ${ }^{15}$

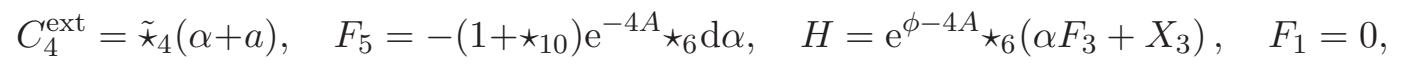

where $A, \alpha, \phi$ are functions on the internal space, $a$ is an integration constant corresponding to a gauge freedom, and $X_{3}$ is an a priori unknown 3-form satisfying $\mathrm{d} X_{3}=0$. One can check that this ansatz follows from the form equations of motion and the requirement that the non-compact part of space-time be maximally symmetric, if only sources with $p=3$ are present.

As in the examples discussed in section 4, the flux-dependent terms $\mathcal{F}(c)$ in (3.33) can now be simplified by a convenient choice of the parameter $c$. To see this recall that the relevant fluxes in the present case are

$$
H^{b} \quad \text { and } \quad F_{7}^{b}
$$

and thus (3.34) reduces to

$$
\mathcal{F}(c)=-c H^{b} \wedge\left[\mathrm{e}^{-\phi} \star_{10} H+F_{3} \wedge C_{4}^{\text {ext }}\right]+(1+c) F_{7}^{b} \wedge F_{3} .
$$

Using (5.2), we find that the first expression on the right-hand side of (5.4) cancels out for $a=0$ except for a term $\sim X_{3}$. The second term in (5.4) can be set to zero by the choice $c=-1$, yielding ${ }^{16}$

$$
\mathcal{F}(-1)=-\tilde{\star}_{4} 1 \wedge H^{b} \wedge X_{3}
$$

We will argue below that, upon a certain choice for the UV boundary conditions of the three-form field strengths, the integral of (5.5) gives a contribution to the cosmological constant in (3.33) that is negligible compared to the contribution from the anti-D3-brane source terms.

Keeping the flux term for the moment, we can substitute (5.5) into (3.33) and write

$$
\begin{aligned}
\Lambda^{\text {class }} & =\frac{1}{4 v \mathcal{V}}\left(S_{\mathrm{DBI}}^{(3)}+S_{\mathrm{CS}}^{(3)}\right)+\frac{1}{4 v \mathcal{V}} \int \mathcal{F}(-1) \\
& =\frac{1}{4 v \mathcal{V}} \mu_{3} \int\left(-\tilde{\star}_{4} \mathrm{e}^{4 A}-C_{4}^{\mathrm{ext}}\right) \wedge \sigma\left(\delta_{6}^{(\overline{\mathrm{D} 3})}\right)+\frac{1}{16 v \mathcal{V}} \mu_{3} \int\left(\tilde{\star}_{4} \mathrm{e}^{4 A}-C_{4}^{\mathrm{ext}}\right) \wedge \sigma\left(\delta_{6}^{(\mathrm{O} 3)}\right)
\end{aligned}
$$

\footnotetext{
${ }^{15}$ If one no longer assumes the BPS condition of section 4.1, the function $\alpha$ need not be related to the warp factor, and $X_{3}$ may be non-vanishing.

${ }^{16}$ To be precise, one finds that the integrated dilaton equation implies $-\int H^{b} \wedge\left[\mathrm{e}^{-\phi} \star_{10} H+F_{3} \wedge C_{4}^{\text {ext }}\right]+$ $\int F_{7}^{b} \wedge F_{3}=0$ in absence of sources with $p \neq 3$, such that $\int \mathcal{F}(c)=-\int \tilde{\star}_{4} 1 \wedge H^{b} \wedge X_{3}$ actually holds for any choice of $c$. This is consistent with the fact that also the source part of (3.33) is independent of $c$ for $p=3$. Thus the value of $\Lambda^{\text {class }}$ is uniquely determined by (3.33) as it should be.
} 


$$
-\frac{1}{4 v \mathcal{V}} \int \tilde{\star}_{4} 1 \wedge H^{b} \wedge X_{3}
$$

where we have spelled out the contributions of the localized sources. Note that the O3plane charge is $\frac{1}{4}$ of the $\overline{\mathrm{D} 3}$-brane charge $\mu_{3}$, where $\mu_{3}>0$ in our conventions. Evaluating the above equation, we find that the total cosmological constant (5.1) is given by

$$
\Lambda=-\frac{1}{4 \mathcal{V}} N_{\overline{\mathrm{D} 3}} \mu_{3}\left(\mathrm{e}^{4 A_{0}}+\alpha_{0}\right)+\frac{1}{16 \mathcal{V}} N_{\mathrm{O} 3} \mu_{3}\left(\mathrm{e}^{4 A_{*}}-\alpha_{*}\right)-\frac{1}{4 \mathcal{V}} \int_{\mathcal{M}^{(6)}} H^{b} \wedge X_{3}+\Lambda^{\mathrm{np}}
$$

where $A_{0}, \alpha_{0}$ and $A_{*}, \alpha_{*}$ denote the values of $A, \alpha$ at the positions of the $\overline{\mathrm{D} 3}$-branes and O3-planes, respectively.

\subsection{The argument}

Our goal is now to evaluate (5.7) and relate it to the near-tip behavior of the energy density of the $H$ flux. In order to do so, we make the following assumptions.

1. Topological flux. In the region of the conifold, $F_{3}$ carries a non-trivial topological flux along the directions of a 3 -cycle called the A cycle, $H$ carries a topological flux along the directions of the dual 3-cycle called the B cycle, and all other components of $H$ and $F_{3}$ are exact. This assumption is due to the fact that the deformed conifold is topologically a cone over $S^{2} \times S^{3}$, where the deformation has the effect of replacing the singular apex of the conifold by a finite $S^{3}$ (see e.g. [43, 44]). The deformed conifold therefore has a non-trivial compact 3-cycle along the $S^{3}$ (the A cycle) and a dual, non-compact 3-cycle (the B cycle). We will assume that also in our compact setting the relevant cycles threaded by topological flux are the A cycle and the B cycle, at least in the region of the conifold. Following the literature [9], we then place $F_{3}$ flux along the A cycle and $H$ flux along the $\mathrm{B}$ cycle. On general compact manifolds, there may of course exist additional cycles that are threaded by flux. We will assume, however, that such additional topologically non-trivial terms in $F_{3}$ and $H$ only become relevant deep in the UV, i.e., far away from the anti-D3-brane.

2. IR boundary conditions. The $\overline{\mathrm{D} 3}$-brane locally deforms the geometry as it would do in flat space. This implies in particular that the warp factor goes to zero in the vicinity of the $\overline{\mathrm{D} 3}$-brane as it usually does,

$$
\mathrm{e}^{2 A} \rightarrow 0
$$

It also implies that we can locally approximate the internal geometry by

$$
g_{m n} \approx \mathrm{e}^{-2 A} \tilde{g}_{m n}
$$

at leading order in an expansion around the distance $r$ to the brane, with $\tilde{g}_{m n}$ regular (in suitable coordinates).

This is a standard assumption discussed recently e.g. in [20,21] for the case of partially smeared $\overline{\mathrm{D} 3}$-branes. In an analogous setting, it was verified explicitly in [24] for the 
toy model with $\overline{\mathrm{D} 6}$-branes discussed in section 4.2 , where both the warp factor and the internal metric indeed diverge exactly as they would do in the corresponding flat space solution [35] at leading order in the distance parameter $r$. It would be interesting to carry out a similar derivation as in [24] also for the $\overline{\mathrm{D} 3}$-branes considered here, but this is beyond the scope of the present paper (see also [20,21] for an analogous discussion of partially smeared $\overline{\mathrm{D} 3}$-branes in the non-compact Klebanov-Strassler solution).

In order that the unperturbed deformed conifold metric $\tilde{g}_{m n}$ shrinks smoothly at the tip, we furthermore expect that the energy density of $F_{3}$ along the A cycle contracted with $\tilde{g}_{m n}$ does not vanish at the tip:

$$
\mathrm{e}^{\phi}\left|\tilde{F}_{3}^{A}\right|^{2} \neq 0
$$

where the superscript denotes the component of $F_{3}$ along the A cycle. ${ }^{17}$ This is motivated by the fact that the energy density of $F_{3}^{A}$ is non-vanishing and prevents the A cycle from collapsing at the tip of the deformed conifold before the perturbation by the $\overline{\mathrm{D} 3}$-branes [9]. Using the results of [21], one can verify that (5.10) indeed holds for the case of partially smeared $\overline{\mathrm{D} 3}$-branes.

3. UV boundary conditions. The boundary conditions for the O3-planes in the UV far away from the $\overline{\mathrm{D} 3}$-branes are approximately the standard BPS boundary conditions,

$$
\alpha_{*} \approx \mathrm{e}^{4 A_{*}},
$$

up to small corrections such that the O3-plane term in (5.7) is negligible compared to the other terms. To justify this, recall that in the GKP setup without the $\overline{\mathrm{D} 3}$-branes this is the usual BPS behavior that does not lead to a contribution to the cosmological constant. When a large flux background with a large number of O3-planes of this type is then perturbed by a small number of $\overline{\mathrm{D} 3}$-branes at the tip of a warped throat, the $\overline{\mathrm{D} 3}$-branes will give a small direct contribution to the cosmological constant due to their tree-level brane action (see below). One might however wonder whether the $\overline{\mathrm{D}}$-brane backreaction on the geometry and the fields could also distort the relation (5.11) near the O3-planes, such that now also the O3-planes would contribute significantly to the vacuum energy. However, this backreaction effect would be of higher order in the small perturbation from the redshifted $\overline{\mathrm{D} 3}$-branes and should thus be negligible compared to the direct contribution from the $\overline{\mathrm{D} 3}$-brane source terms. This is analogous to the usual assumption of BPS asymptotics in the UV imposed in non-compact treatments of brane backreaction (e.g. [20, 25]). It would be an interesting extension to explicitly compute the boundary conditions at the O-planes, e.g. following the analysis in [24].

Similarly, we also assume that the three-form field strengths approach their unperturbed values and thus become ISD in the UV far away from the $\overline{\mathrm{D} 3}$-branes, which

\footnotetext{
${ }^{17}$ This is not to be confused with the notation of [19, 21], where the superscript in $F_{3}^{A}$ is an index running over all components of $F_{3}$.
} 
implies

$$
X_{3}^{\mathrm{UV}} \approx 0,
$$

again up to corrections that are negligible in (5.7). One might again wonder whether a small deviation from the ISD condition in the UV due to the anti-brane backreaction might be relevant for the value of the cosmological constant. As discussed above, however, it would be very surprising if the effect of such a deviation far away from the $\overline{\mathrm{D} 3}$-branes would not be negligible compared to their direct effect in the IR, so that we will adopt (5.12) as a reasonable assumption.

4. Non-perturbative corrections. Non-perturbative corrections to the effective potential (due to, e.g., Euclidean D3-branes or gaugino condensation on D7-branes) are captured by adding a negative term to the overall cosmological constant, i.e.

$$
\Lambda=\Lambda^{\text {class }}-\left|\Lambda^{\mathrm{np}}\right| .
$$

This assumption consists in fact of two parts: the first is that the non-perturbative effect gives, by itself, rise to a negative contribution to the vacuum energy, and the second is that it does not significantly change the classical contributions. These assumptions are implicit in the construction of [12], where the non-perturbative effects first make the vanishing cosmological constant of the GKP setup negative without significantly changing the classical background fluxes or the vevs and masses of the moduli that are stabilized by these fluxes (the complex structure moduli and the dilaton). Moreover, the subsequent de Sitter uplift due to $\overline{\mathrm{D} 3}$-branes is assumed to happen through their classical source terms only and does in turn not significantly change the vevs and masses of the moduli that are stabilized by the non-perturbative effects (the Kähler moduli). There has also been some progress in describing the above effects from an explicit 10D point of view [45-48]. In [48] it was argued that a non-vanishing gaugino bilinear $\langle\bar{\lambda} \lambda\rangle$ on D7-branes indeed leads to a negative contribution to the $4 \mathrm{D}$ spacetime curvature proportional to $|\langle\bar{\lambda} \lambda\rangle|^{2}$. On the other hand, the backreaction of this on the classical contribution $\Lambda^{\text {class }}$ to the vacuum energy would be only a higher order effect. Similar properties are expected for the non-perturbative corrections due to Euclidean D3-brane instantons.

5. Cosmological constant. The presence of the $\overline{\mathrm{D} 3}$-branes uplifts the solution to a meta-stable de Sitter vacuum such that the total cosmological constant of the solution is positive,

$$
\Lambda>0,
$$

as proposed in [12].

If one makes the above assumptions 1. - 5., our ansatz (5.7) for the cosmological constant drastically simplifies.

Let us at first discuss the flux term in (5.7). Since $X_{3}$ is closed by definition, we can make the ansatz

$$
X_{3}=\beta \omega_{3}^{A}+\mathrm{d} \omega_{2}
$$


in the conifold region. Here $\beta$ is an unknown function of the internal coordinates, $\omega_{2}$ is a 2 -form, and $\omega_{3}^{A}$ is the harmonic 3-form along the A cycle satisfying $\mathrm{d} \omega_{3}^{A}=0$. We have split $X_{3}$ into a part, $\beta \omega_{3}^{A}$, along the A cycle, which can in general be non-exact, and a part, $\mathrm{d} \omega_{2}$, that is not necessarily along the $A$ cycle and has to be exact. ${ }^{18}$ Using $\mathrm{d} X_{3}=\mathrm{d} \omega_{3}^{A}=0$, we find from (5.15) that

$$
\mathrm{d} \beta \wedge \omega_{3}^{A}=0,
$$

which implies that $\beta$ is only a function of the coordinates parametrizing the $S^{3}$ but constant over the remaining directions. We can therefore set $\beta=\beta^{\mathrm{UV}}=0$ without loss of generality, where $\beta^{\mathrm{UV}}$ denotes the value of $\beta$ in the UV region of the warped throat far away from the $\overline{\mathrm{D} 3}$-branes.

The flux term in (5.7) then simplifies as follows. Since, under assumption 1., $H$ only carries a flux along the $\mathrm{B}$ cycle in the conifold region, we find $H^{b} \wedge X_{3}=H^{b} \wedge\left(\beta \omega_{3}^{A}+\mathrm{d} \omega_{2}\right)=$ $H^{b} \wedge \beta^{\mathrm{UV}} \omega_{3}^{A}-\mathrm{d}\left(H^{b} \wedge \omega_{2}\right)$. We can therefore write

$$
\int_{\mathcal{M}^{(6)}} H^{b} \wedge X_{3}=\int_{\mathcal{M}^{(6)}} H^{b} \wedge X_{3}^{\mathrm{UV}}=0
$$

such that the integral is completely determined by the units of $H$ flux present in the compactification and the UV boundary conditions for the three-form field strengths but independent of the IR physics close to the $\overline{\mathrm{D} 3}$-branes.

Using (5.17) together with assumptions 2.- 4., we find that (5.7) reduces to

$$
\Lambda \approx-\frac{1}{4 \mathcal{V}} N_{\overline{\mathrm{D} 3}} \mu_{3} \alpha_{0}-\left|\Lambda^{\mathrm{np}}\right|
$$

up to negligible corrections. From assumption 5. it then follows that

$$
-\frac{1}{4 \mathcal{V}} N_{\overline{\mathrm{D} 3}} \mu_{3} \alpha_{0}>\left|\Lambda^{\mathrm{np}}\right|
$$

which implies that $\alpha_{0}$ must be finite and negative. ${ }^{19}$

It is straightforward to see that this yields a singular energy density of the $H$ flux in the region near the $\overline{\mathrm{D} 3}$-branes. As argued above, we can locally approximate the internal metric as $g_{m n} \approx \mathrm{e}^{-2 A} \tilde{g}_{m n}$, where $\tilde{g}_{m n}$ is regular. Using (5.2), we can then write

$$
\mathrm{e}^{-\phi}|H|^{2}=\mathrm{e}^{\phi-8 A}\left|\alpha F_{3}+X_{3}\right|^{2} \geq \alpha^{2} \mathrm{e}^{-8 A} \mathrm{e}^{\phi}\left|F_{3}^{A}\right|^{2} \approx \alpha^{2} \mathrm{e}^{-2 A} \mathrm{e}^{\phi}\left|\tilde{F}_{3}^{A}\right|^{2}
$$

in the near-brane region, where we have used that the component of $X_{3}$ along $F_{3}^{A}$ vanishes. Since $e^{\phi}\left|\tilde{F}_{3}^{A}\right|^{2}$ is expected to be non-zero at the tip of the conifold, it then follows from (5.8) and $\alpha_{0} \neq 0$ that the energy density of the $H$ flux at least diverges like the inverse of the warp factor,

$$
\mathrm{e}^{-\phi}|H|^{2} \sim \mathrm{e}^{-2 A}
$$

\footnotetext{
${ }^{18}$ Note that, assuming the presence of $F_{3}$ flux along the A cycle, $X_{3}$ is not allowed to have a non-exact component along the $\mathrm{B}$ cycle as follows from the $F_{1}$ equation $\mathrm{e}^{-\phi} H \wedge \star_{10} F_{3}=0$ and the ansatz for $H$ stated in (5.2).

${ }^{19}$ Note that $\alpha$ must change its sign somewhere in between the BPS region around the O3-planes (where $\alpha \approx \mathrm{e}^{4 A}$ ) and the tip of the throat (where $\alpha<0$ ). In the toy model discussed in [3], a similar constraint was used to formulate a topological no-go theorem, which is rederived in our framework in section 4.2.
} 
Assuming a regular dilaton, ${ }^{20}$ the dilaton equation (2.15) furthermore implies that the divergence in the energy density of $H$ must be canceled by a divergent term in the energy density of $F_{3}$. We thus find that the energy densities of $H$ and $F_{3}$ diverge at least as ${ }^{21}$

$$
\mathrm{e}^{-\phi}|H|^{2} \sim \mathrm{e}^{-2 A}, \quad \mathrm{e}^{\phi}\left|F_{3}\right|^{2} \sim \mathrm{e}^{-2 A} .
$$

Note that, due to its global nature, the argument is independent of most details of the bulk dynamics and does therefore not require simplifications such as a partial smearing of the branes or a linearization of the equations of motion. Under the assumptions discussed above, it holds for fully localized branes that backreact on the full non-linear equations of motion.

\section{Conclusion}

We have shown how the 10D equations of motion for classical type II supergravity can be combined to give a surprisingly simple expression for the cosmological constant in terms of the classical near-source behavior of the supergravity fields and a contribution from topologically non-trivial background fluxes. The derivation relies on no specific assumptions on the compactification manifold, but it holds only for maximally symmetric spacetimes of dimension four or more. In simple examples, the flux contribution can be chosen to be zero, and the expression reduces to contributions that have support only on localized sources. This extends the recent work [5] to general brane and flux setups. We checked our result against some well-understood examples of flux compactifications and found agreement with all expectations. We specified the assumptions that are required to apply our result also to de Sitter uplifts from $\overline{\mathrm{D} 3}$-branes in warped throats and showed that this would then indicate the presence of a singular $H$ and $F_{3}$ energy density at the $\overline{\mathrm{D} 3}$-brane similar to what has been reported in recent studies of the same setup [14-17, 20]. Although our analysis does not clarify the physical meaning of this singularity (see $[22,23,49]$ for a recent conjecture), it indicates that it is unlikely a mere artifact of approximations such as partial smearing or linearized field equations, which we do not use.

It should be interesting to apply our general result also to other aspects of string compactifications.

\section{Acknowledgments}

The authors would like to thank Gary Shiu, Yoske Sumitomo and Thomas Van Riet for useful discussions. D. J. would also like to thank the organizers of the workshop "Brane backreaction, fluxes and meta-stable vacua in string theory" at Uppsala Universitet for hospitality. This work was supported by the German Research Foundation (DFG) within the Cluster of Excellence "QUEST".

\footnotetext{
${ }^{20}$ If the dilaton diverges at the brane even though it does not directly couple to it, $\mathrm{e}^{-\phi}|H|^{2}$ would still diverge, but the dilaton equation would not necessarily imply that $\mathrm{e}^{\phi}\left|F_{3}\right|^{2}$ also diverges.

${ }^{21}$ Evaluating this equation for the case of partially smeared $\overline{\mathrm{D} 3}$-branes, we recover the result of [21], where it was shown that $\mathrm{e}^{2 A} \sim \tau^{1 / 2}$ and $\mathrm{e}^{-\phi}|H|^{2} \sim \mathrm{e}^{\phi}\left|F_{3}\right|^{2} \sim \tau^{-1 / 2}$ near the tip of the conifold and $\tau$ is the radial coordinate transverse to the branes in the conventions of [21].
} 


\section{A Explicit manipulations of the equations of motion}

Here we present an alternative derivation of our main result (3.33), which only uses the equations of motion. We first consider the Bianchi identity (2.14) for the internal RR field strength $F_{8-p}^{\text {int }}$ and multiply by $\sigma\left(C_{p+1}^{\text {ext }}\right)$,

$$
\begin{aligned}
& 0=-\alpha \sigma\left(C_{p+1}^{\text {ext }}\right) \wedge\left\langle\mathrm{d}_{-H} F^{\text {int }}+j\right\rangle_{9-p} \\
& =\mathrm{d}\left[\sigma\left(C_{p+1}^{\text {ext }}\right) \wedge F_{8-p}^{\text {int }}\right]+\alpha\left\langle\sigma\left(\mathrm{d}_{-H} C^{\text {ext }}\right)\right\rangle_{p+2} \wedge F_{8-p}^{\text {int }}+\alpha \sigma\left(H \wedge C_{p-1}^{\text {ext }}\right) \wedge F_{8-p}^{\text {int }} \\
& +\alpha \sigma\left(C_{p+1}^{\mathrm{ext}}\right) \wedge H \wedge F_{6-p}^{\mathrm{int}}-\alpha \sigma\left(C_{p+1}^{\mathrm{ext}}\right) \wedge j_{9-p} \\
& =\mathrm{d}\left[\sigma\left(C_{p+1}^{\text {ext }}\right) \wedge F_{8-p}^{\text {int }}\right]+\alpha \sigma\left\langle F^{\text {ext }}-\mathrm{e}^{B} \wedge F^{b}\right\rangle_{p+2} \wedge F_{8-p}^{\text {int }}-\alpha \sigma\left(F_{8-p}^{\text {int }}\right) \wedge H \wedge C_{p-1}^{\text {ext }} \\
& +\alpha \sigma\left(F_{6-p}^{\mathrm{int}}\right) \wedge H \wedge C_{p+1}^{\mathrm{ext}}+\alpha \sigma\left(j_{9-p}\right) \wedge C_{p+1}^{\mathrm{ext}} \\
& =\mathrm{d}\left[\sigma\left(C_{p+1}^{\mathrm{ext}}\right) \wedge F_{8-p}^{\mathrm{int}}\right]-\mathrm{e}^{(p-3) \phi / 2} \star_{10}\left|F_{8-p}^{\mathrm{int}}\right|^{2}+\left\langle\mathrm{e}^{B} \wedge F^{b}\right\rangle_{p+2} \wedge \sigma\left(F_{8-p}^{\mathrm{int}}\right) \\
& -H \wedge C_{p-1}^{\text {ext }} \wedge \sigma\left(F_{8-p}^{\text {int }}\right)+H \wedge C_{p+1}^{\text {ext }} \wedge \sigma\left(F_{6-p}^{\text {int }}\right)-C_{p+1}^{\text {ext }} \wedge \sigma\left(j_{9-p}\right) .
\end{aligned}
$$

Here we have introduced the constant $\alpha$ which equals +1 for type IIA and -1 for type IIB supergravity. Multiplying the $H$ equation of motion (2.16) by $B$ yields

$$
\begin{aligned}
0= & 2 B \wedge \mathrm{d}\left(\mathrm{e}^{-\phi} \star_{10} H\right)-\alpha\langle B \wedge \sigma(F) \wedge F\rangle_{10} \\
= & 2 B \wedge \mathrm{d}\left(\mathrm{e}^{-\phi} \star_{10} H\right)-2 \alpha\left\langle B \wedge \sigma\left(F^{\text {int }}\right) \wedge F^{\text {ext }}\right\rangle_{10} \\
= & 2 \mathrm{~d}\left\langle\mathrm{e}^{-\phi} B \wedge \star_{10} H-B \wedge \sigma\left(F^{\text {int }}\right) \wedge C^{\text {ext }}\right\rangle_{9}-2\left(H-H^{b}\right) \wedge\left(\mathrm{e}^{-\phi} \star_{10} H\right) \\
& +2\left\langle\mathrm{~d}_{H}\left(B \wedge \sigma\left(F^{\text {int }}\right)\right) \wedge C^{\text {ext }}-\alpha B \wedge \sigma\left(F^{\text {int }}\right) \wedge \mathrm{e}^{B} \wedge F^{b}\right\rangle_{10} \\
= & 2 \mathrm{~d}\left\langle\mathrm{e}^{-\phi} B \wedge \star_{10} H-B \wedge \sigma\left(F^{\text {int }}\right) \wedge C^{\text {ext }}\right\rangle_{9}-2\left(H-H^{b}\right) \wedge\left(\mathrm{e}^{-\phi} \star_{10} H-\left\langle\sigma\left(F^{\text {int }}\right) \wedge C^{\text {ext }}\right\rangle_{7}\right) \\
& -2\left\langle-B \wedge C^{\text {ext }} \wedge \sigma(j)+\mathrm{e}^{B} \wedge F^{b} \wedge B \wedge \sigma\left(F^{\text {int }}\right)\right\rangle_{10} .
\end{aligned}
$$

Notice in above equation that $F_{6}^{\text {int }}$ never appears since $F^{\text {int }}$ is everywhere multiplied by either $B$ or $H$, which must both be purely internal in a maximally symmetric compactification to $d \geq 4$ dimensions. We now take the combination $(1+(p-3) c / 2)$ times (A.1) plus $c / 2$ times (A.2) and sum over $p$. Substituting the definition of $j$ from section 2, this yields

$$
\begin{aligned}
0= & \sum_{3 \leq p}\left(1+\frac{p-3}{2} c\right)\left\{-\mathrm{e}^{(p-3) \phi / 2} \star_{10}\left|F_{8-p}^{\mathrm{int}}\right|^{2}-C_{p+1}^{\mathrm{ext}} \wedge \sigma\left(j_{9-p}\right)\right\} \\
& +c\left\langle\mathrm{e}^{-\phi} \star_{10}|H|^{2}+B \wedge C^{\mathrm{ext}} \wedge \sigma(j)\right\rangle_{10}-\Sigma(c)+\text { total derivatives } \\
= & \sum_{3 \leq p}\left(1+\frac{p-3}{2} c\right)\left(-\mathrm{e}^{(p-3) \phi / 2} \star_{10}\left|F_{8-p}^{\mathrm{int}}\right|^{2}-S_{\mathrm{CS}}^{(p)}\right) \\
& +c \mathrm{e}^{-\phi} \star_{10}|H|^{2}-\Sigma(c)+\text { total derivatives, }
\end{aligned}
$$

where $c$ is a free parameter. We also introduced the shorthand

$$
\Sigma(c)=-\sum_{2 \leq p}\left(1+\frac{p-3}{2} c\right) F_{p+2}^{b} \wedge\left\langle\mathrm{e}^{B} \wedge \sigma\left(F^{\mathrm{int}}\right)\right\rangle_{8-p}+\left(1-\frac{1}{2} c\right) F_{4}^{b} \wedge \sigma\left(F_{6}^{\mathrm{int}}\right)
$$




$$
-c H^{b} \wedge\left(\mathrm{e}^{-\phi} \star H-\left\langle\sigma\left(F^{\mathrm{int}}\right) \wedge C^{\mathrm{ext}}\right\rangle_{7}\right)
$$

where we have combined all terms that depend on background fluxes to simplify our notation.

The trace of the external components of the (trace-reversed) Einstein equation reads

$$
\frac{4}{d} R_{d}=-\frac{1}{2} \mathrm{e}^{-\phi}|H|^{2}+\sum_{3 \leq p} \frac{p-7}{4}\left(\mathrm{e}^{(p-3) \phi / 2}\left|F_{8-p}^{\text {int }}\right|^{2} \pm \mu_{p} \mathrm{e}^{(p-3) \phi / 4} \delta(\Sigma)\right)+\frac{5}{4} \mathrm{e}^{\phi / 2}\left|F_{4}^{\text {ext }}\right|^{2},
$$

where the upper sign is for D-branes and the lower sign for O-planes and we have used $\left|F_{5}^{\text {ext }}\right|^{2}=-\left|F_{5}^{\text {int }}\right|^{2}$ to rewrite the spacetime-filling part of $\left|F_{5}\right|^{2}$. Note that spacetime-filling $F_{4}$ flux can only be present for $d=4$ in type IIA supergravity, while $F_{5}$ flux can be present for $d=4$ or $d=5$ in type IIB supergravity.

The dilaton equation (2.15) yields

$$
0=-\nabla^{2} \phi-\frac{1}{2} \mathrm{e}^{-\phi}|H|^{2}+\sum_{3 \leq p} \frac{p-3}{4}\left(\mathrm{e}^{(p-3) \phi / 2}\left|F_{8-p}^{\mathrm{int}}\right|^{2} \pm \mu_{p} \mathrm{e}^{(p-3) \phi / 4} \delta(\Sigma)\right)+\frac{1}{4} \mathrm{e}^{\phi / 2}\left|F_{4}^{\text {ext }}\right|^{2} .
$$

Combining (A.5) and (A.6), we find

$$
\begin{aligned}
\frac{4}{d} R_{d}= & c \mathrm{e}^{-\phi}|H|^{2}+\sum_{3 \leq p}\left(1+\frac{p-3}{2} c\right)\left(-\mathrm{e}^{(p-3) \phi / 2}\left|F_{8-p}^{\text {int }}\right|^{2} \mp \mu_{p} \mathrm{e}^{(p-3) \phi / 4} \delta(\Sigma)\right) \\
& +\left(1-\frac{c}{2}\right) \mathrm{e}^{\phi / 2}\left|F_{4}^{\text {ext }}\right|^{2}+\text { total derivatives. }
\end{aligned}
$$

Finally, we can combine (A.7) with (A.3) to get

$$
\begin{aligned}
\frac{4}{d} \star_{10} R_{d}= & \sum_{3 \leq p}\left(1+\frac{p-3}{2} c\right)\left(\mp \star_{10} \mu_{p} \mathrm{e}^{(p-3) \phi / 4} \delta(\Sigma)+S_{\mathrm{CS}}^{(p)}\right)+\mathcal{F}(c) \\
& + \text { total derivatives, }
\end{aligned}
$$

where we defined

$$
\mathcal{F}(c)=\Sigma(c)-\left(1-\frac{c}{2}\right) F_{4}^{b} \wedge \sigma\left(F_{6}^{\text {int }}\right)
$$

and used $\mathrm{e}^{\phi / 2} \star_{10} F_{4}^{\text {ext }}=-\sigma\left(F_{6}^{\text {int }}\right)$, which follows from the duality relations (2.4). Integrating over ten-dimensional space and using (2.11), we get rid of all total derivative terms and find

$$
\frac{8 v \mathcal{V}}{d-2} \Lambda=\sum_{p}\left(1+\frac{p-3}{2} c\right)\left[S_{\mathrm{DBI}}^{(p)}+S_{\mathrm{CS}}^{(p)}\right]+\int \mathcal{F}(c)
$$

with the volume factors $v$ and $\mathcal{V}$ defined as in (2.13).

\section{References}

[1] M.R. Douglas and R. Kallosh, Compactification on negatively curved manifolds, JHEP 06 (2010) 004 [arXiv:1001.4008] [INSPIRE]. 
[2] J. Blaback, U.H. Danielsson, D. Junghans, T. Van Riet, T. Wrase and M. Zagermann, Smeared versus localised sources in flux compactifications, JHEP 12 (2010) 043 [arXiv: 1009.1877] [INSPIRE].

[3] J. Blaback, U.H. Danielsson, D. Junghans, T. Van Riet, T. Wrase and M. Zagermann, The problematic backreaction of SUSY-breaking branes, JHEP 08 (2011) 105 [arXiv:1105.4879] [INSPIRE].

[4] Y. Aghababaie, C. Burgess, J.M. Cline, H. Firouzjahi, S. Parameswaran, F. Quevedo, G. Tasinato and I. Zavala, Warped brane worlds in six-dimensional supergravity, JHEP 09 (2003) 037 [hep-th/0308064] [INSPIRE].

[5] C. Burgess, A. Maharana, L. van Nierop, A. Nizami and F. Quevedo, On brane back-reaction and de Sitter solutions in higher-dimensional supergravity, JHEP 04 (2012) 018 [arXiv: 1109.0532] [INSPIRE].

[6] F.F. Gautason, D. Junghans and M. Zagermann, On cosmological constants from $\alpha^{\prime}$-corrections, JHEP 06 (2012) 029 [arXiv: 1204.0807] [INSPIRE].

[7] E. Witten, Dimensional reduction of superstring models, Phys. Lett. B 155 (1985) 151 [INSPIRE].

[8] C. Burgess, A. Font and F. Quevedo, Low-energy effective action for the superstring, Nucl. Phys. B 272 (1986) 661 [inSPIRE].

[9] I.R. Klebanov and M.J. Strassler, Supergravity and a confining gauge theory: duality cascades and $\chi \mathrm{SB}$ resolution of naked singularities, JHEP 08 (2000) 052 [hep-th/0007191] [INSPIRE].

[10] S.B. Giddings, S. Kachru and J. Polchinski, Hierarchies from fluxes in string compactifications, Phys. Rev. D 66 (2002) 106006 [hep-th/0105097] [INSPIRE].

[11] S. Kachru, J. Pearson and H.L. Verlinde, Brane/flux annihilation and the string dual of a nonsupersymmetric field theory, JHEP 06 (2002) 021 [hep-th/0112197] [INSPIRE].

[12] S. Kachru, R. Kallosh, A.D. Linde and S.P. Trivedi, De Sitter vacua in string theory, Phys. Rev. D 68 (2003) 046005 [hep-th/0301240] [INSPIRE].

[13] O. DeWolfe, S. Kachru and M. Mulligan, A gravity dual of metastable dynamical supersymmetry breaking, Phys. Rev. D 77 (2008) 065011 [arXiv:0801.1520] [INSPIRE].

[14] P. McGuirk, G. Shiu and Y. Sumitomo, Non-supersymmetric infrared perturbations to the warped deformed conifold, Nucl. Phys. B 842 (2011) 383 [arXiv:0910.4581] [INSPIRE].

[15] I. Bena, M. Graña and N. Halmagyi, On the existence of meta-stable vacua in Klebanov-Strassler, JHEP 09 (2010) 087 [arXiv:0912.3519] [INSPIRE].

[16] I. Bena, G. Giecold, M. Graña, N. Halmagyi and S. Massai, On metastable vacua and the warped deformed conifold: analytic results, Class. Quant. Grav. 30 (2013) 015003 [arXiv: 1102.2403] [INSPIRE].

[17] I. Bena, G. Giecold, M. Graña, N. Halmagyi and S. Massai, The backreaction of $\overline{\mathrm{D} 3}$ branes on the Klebanov-Strassler geometry, JHEP 06 (2013) 060 [arXiv:1106.6165] [INSPIRE].

[18] A. Dymarsky, On gravity dual of a metastable vacuum in Klebanov-Strassler theory, JHEP 05 (2011) 053 [arXiv: 1102.1734] [INSPIRE].

[19] S. Massai, A comment on anti-brane singularities in warped throats, arXiv:1202.3789 [INSPIRE]. 
[20] I. Bena, M. Graña, S. Kuperstein and S. Massai, $\overline{\mathrm{D} 3}$ 's - singular to the bitter end, Phys. Rev. D 87 (2013) 106010 [arXiv:1206.6369] [InSPIRE].

[21] I. Bena, M. Graña, S. Kuperstein and S. Massai, Polchinski-Strassler does not uplift Klebanov-Strassler, arXiv:1212.4828 [INSPIRE].

[22] I. Bena, A. Buchel and O.J. Dias, Horizons cannot save the landscape, Phys. Rev. D 87 (2013) 063012 [arXiv:1212.5162] [InSPIRE].

[23] J. Blaback, U.H. Danielsson and T. Van Riet, Resolving anti-brane singularities through time-dependence, JHEP 02 (2013) 061 [arXiv:1202.1132] [INSPIRE].

[24] J. Blaback, U.H. Danielsson, D. Junghans, T. Van Riet, T. Wrase and M. Zagermann, (Anti-)brane backreaction beyond perturbation theory, JHEP 02 (2012) 025 [arXiv: 1111.2605] [INSPIRE].

[25] I. Bena, D. Junghans, S. Kuperstein, T. Van Riet, T. Wrase and M. Zagermann, Persistent anti-brane singularities, JHEP 10 (2012) 078 [arXiv: 1205.1798] [INSPIRE].

[26] P. Koerber, Lectures on generalized complex geometry for physicists, Fortsch. Phys. 59 (2011) 169 [arXiv:1006.1536] [INSPIRE].

[27] E. Bergshoeff, R. Kallosh, T. Ortín, D. Roest and A. Van Proeyen, New formulations of $D=10$ supersymmetry and D8-O8 domain walls, Class. Quant. Grav. 18 (2001) 3359 [hep-th/0103233] [INSPIRE].

[28] C.G. Callan Jr., C. Lovelace, C. Nappi and S. Yost, String loop corrections to $\beta$-functions, Nucl. Phys. B 288 (1987) 525 [inSPIRE].

[29] E. Cremmer, H. Lü, C. Pope and K. Stelle, Spectrum generating symmetries for BPS solitons, Nucl. Phys. B 520 (1998) 132 [hep-th/9707207] [INSPIRE].

[30] P.G. Freund and M.A. Rubin, Dynamics of dimensional reduction, Phys. Lett. B 97 (1980) 233 [InSPIRE].

[31] K. Dasgupta, G. Rajesh and S. Sethi, $M$ theory, orientifolds and G-flux, JHEP 08 (1999) 023 [hep-th/9908088] [INSPIRE].

[32] S. Gukov, C. Vafa and E. Witten, CFT's from Calabi-Yau four folds, Nucl. Phys. B 584 (2000) 69 [Erratum ibid. B 608 (2001) 477] [hep-th/9906070] [INSPIRE].

[33] K. Becker and M. Becker, M theory on eight manifolds, Nucl. Phys. B 477 (1996) 155 [hep-th/9605053] [INSPIRE].

[34] B.R. Greene, K. Schalm and G. Shiu, Warped compactifications in $M$ and F theory, Nucl. Phys. B 584 (2000) 480 [hep-th/0004103] [INSPIRE].

[35] B. Janssen, P. Meessen and T. Ortín, The D8-brane tied up: string and brane solutions in massive type IIA supergravity, Phys. Lett. B 453 (1999) 229 [hep-th/9901078] [INSPIRE].

[36] C. Caviezel, P. Koerber, S. Körs, D. Lüst, T. Wrase and M. Zagermann, On the cosmology of type IIA compactifications on SU(3)-structure manifolds, JHEP 04 (2009) 010 [arXiv:0812.3551] [INSPIRE].

[37] U.H. Danielsson, S.S. Haque, P. Koerber, G. Shiu, T. Van Riet and T. Wrase, De Sitter hunting in a classical landscape, Fortsch. Phys. 59 (2011) 897 [arXiv:1103.4858] [INSPIRE].

[38] O. DeWolfe, A. Giryavets, S. Kachru and W. Taylor, Type IIA moduli stabilization, JHEP 07 (2005) 066 [hep-th/0505160] [INSPIRE]. 
[39] B.S. Acharya, F. Benini and R. Valandro, Fixing moduli in exact type IIA flux vacua, JHEP 02 (2007) 018 [hep-th/0607223] [INSPIRE].

[40] T. Banks and K. van den Broek, Massive IIA flux compactifications and U-dualities, JHEP 03 (2007) 068 [hep-th/0611185] [INSPIRE].

[41] F. Saracco and A. Tomasiello, Localized O6-plane solutions with Romans mass, JHEP 07 (2012) 077 [arXiv: 1201.5378] [INSPIRE].

[42] J. McOrist and S. Sethi, M-theory and type IIA flux compactifications, JHEP 12 (2012) 122 [arXiv: 1208.0261] [INSPIRE].

[43] P. Candelas and X.C. de la Ossa, Comments on conifolds, Nucl. Phys. B 342 (1990) 246 [INSPIRE].

[44] R. Minasian and D. Tsimpis, On the geometry of nontrivially embedded branes, Nucl. Phys. B 572 (2000) 499 [hep-th/9911042] [INSPIRE].

[45] P. Koerber and L. Martucci, From ten to four and back again: how to generalize the geometry, JHEP 08 (2007) 059 [arXiv:0707.1038] [inSPIRE].

[46] D. Baumann, A. Dymarsky, S. Kachru, I.R. Klebanov and L. McAllister, D3-brane potentials from fluxes in AdS/CFT, JHEP 06 (2010) 072 [arXiv: 1001.5028] [InSPIRE].

[47] A. Dymarsky and L. Martucci, D-brane non-perturbative effects and geometric deformations, JHEP 04 (2011) 061 [arXiv: 1012.4018] [INSPIRE].

[48] B. Heidenreich, L. McAllister and G. Torroba, Dynamic SU(2) structure from seven-branes, JHEP 05 (2011) 110 [arXiv:1011.3510] [INSPIRE].

[49] I. Bena, J. Blaback, U. Danielsson and T. Van Riet, Antibranes don't go black, Phys. Rev. D 87 (2013) 104023 [arXiv:1301.7071] [INSPIRE]. 